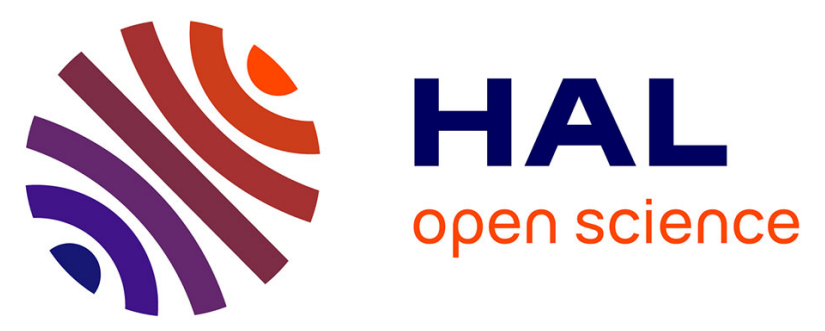

\title{
Solar Lyman alpha changes and related hydrogen density distribution at the Earth's exobase (1969-1970)
}

A. Vidal-Madjar, Jacques Emile Blamont, B. Phissamay

\section{To cite this version:}

A. Vidal-Madjar, Jacques Emile Blamont, B. Phissamay. Solar Lyman alpha changes and related hydrogen density distribution at the Earth's exobase (1969-1970). Journal of Geophysical Research Space Physics, 1973, 78 (7), pp.1115-1144. 10.1029/JA078i007p01115 . insu-03590190

\section{HAL Id: insu-03590190 \\ https://hal-insu.archives-ouvertes.fr/insu-03590190}

Submitted on 26 Feb 2022

HAL is a multi-disciplinary open access archive for the deposit and dissemination of scientific research documents, whether they are published or not. The documents may come from teaching and research institutions in France or abroad, or from public or private research centers.
L'archive ouverte pluridisciplinaire HAL, est destinée au dépôt et à la diffusion de documents scientifiques de niveau recherche, publiés ou non, émanant des établissements d'enseignement et de recherche français ou étrangers, des laboratoires publics ou privés.

$$
\text { Copyright }
$$




\title{
Solar Lyman Alpha Changes and Related Hydrogen Density Distribution at the Earth's Exobase (1969-1970)
}

\author{
A. Vidal-Madjar \\ Laboratoire de Physique Stellaire et Planétaire \\ Centre National de la Recherche Scientifique, Verrières-le-Buisson, France \\ J. E. Blamont and B. Phissamay \\ Service d'Aéronomie, Centre National de la Recherche Scientifique \\ Verrières-le-Buisson, France
}

\begin{abstract}
The University of Paris experiment, operating on board the Oso 5 spacecraft since January 22, 1969, observes the solar Lyman $\alpha$ line integrated over the whole solar disk. The hydrogen and deuterium resonance cells yield information about the solar flux at the center of the line and on the blue wing. The center of the solar line as observed from the spacecraft is strongly absorbed by the earth's geocorona. Therefore the hydrogen resonance data contain solar, as well as geocoronal, information. Comparison of the data with sophisticated exospheric models gives the following principal results: (1) A high correlation ( 0.87 coefficient) exists between the total Lyman $\alpha$ line flux and the center line flux. A 30\% variation in the total flux corresponds to a 47\% variation in the central flux. The average fluxes over 1969 and 1970 are $3 \times 10^{11} \mathrm{ph} \mathrm{cm}^{-2} \mathrm{sec}^{-1}$ total flux and $5 \times 10^{11} \mathrm{ph} \mathrm{cm}^{-2} \mathrm{sec}^{-1} \mathrm{~A}^{-1}$ central flux. (2) The absolute value of the hydrogen density at $500-\mathrm{km}$ altitude (independent of the experiment's calibration) is of the order of $5 \times 10^{4}$ atoms $\mathrm{cm}^{-3}$ on the day side and $9 \times 10^{4}$ atoms $\mathrm{cm}^{-3}$ on the night side. (3) The earth's hydrogen exobase is controlled by the zero net ballistic flux condition (i.e., the outgoing ballistic flux is everywhere equal to the incoming ballistic flux). This is why an average ratio of 1.7 was found between the maximum and minimum densities at the exobase. A discrepancy appeared between the absolute values of the hydrogen density measured by this experiment and the corresponding absolute values predicted by the model of G. Kockarts and M. Nicolet (1963). In order to fit our data, the values of the model should be increased to the following hydrogen densities at an altitude of $100 \mathrm{~km}: 2.3 \times 10^{7}$ atoms $\mathrm{cm}^{-3}$ for low solar activity (Zurich number $R z \sim 60$ ) $; 2.5 \times 10^{7}$ atoms $\mathrm{cm}^{-3}$ for mean solar activity $(R z \sim 120)$; and $4 \times 10^{7}$ atoms $\mathrm{cm}^{-8}$ for high solar activity $(R z \sim 180)$.
\end{abstract}

The solar Lyman $\alpha$ line has been monitored since January 22, 1969, by the University of Paris experiment [Blamont and Vidal-Madjar, 1971]. This experiment measures over the entire solar disk three different types of data, shown in Figure 1: (1) the flux of the solar spectrum integrated over $100 \mathrm{~A}$, centered at Lyman $\alpha$ and measured in two separate channels (one called the hydrogen channel and the other the deuterium channel); (2) the solar flux in a $10^{-2}-\mathrm{A}$ bandpass at the center of the Lyman $\alpha$ line (resonance of the hydrogen atom at $1215.664 \mathrm{~A}$ ); and (3) the solar flux in a $10^{-2}-\mathrm{A}$ bandpass placed on the blue wing of the Lyman $\alpha$ line (resonance of the deuterium atom at $1215.334 \mathrm{~A}$ ). Since the resonance deuterium

Copyright (a) 1973 by the American Geophysical Union. data are still too complicated to warrant a clear interpretation because of the presence of hydrogen in the deuterium resonance cell, we will restrict the present analysis to the first two types of data.

The two channels measuring the solar flux in the 100-A bandpass centered at Lyman $\alpha$ are very sensitive to the spacecraft's attitude, and, as is shown in Figure 2, they show rapid variations which are, of course, not related to solar activity variations. On the other hand, the resonance hydrogen data show very smooth day to day changes, generally well related to variations in solar activity. The variations of the resonance hydrogen data during one orbit are very stable, as far as the general shape of this variation is concerned.

In Figure 3, several orbits plotted as a func- 


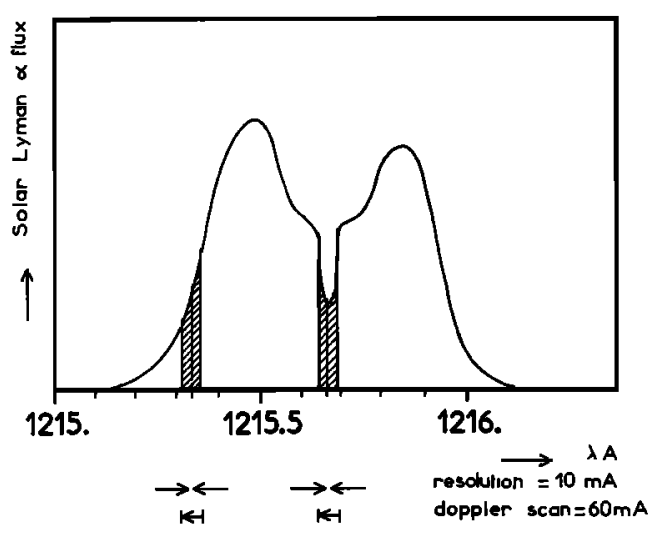

Fig. 1. The solar Lyman a profile.

tion of the earth-satellite earth-sun angle show that beyond $80^{\circ}$ there is a decrease in the number of counts owing to the geocoronal atomic hydrogen absorption. The red wing of the geocoronal absorption core is fainter than the blue wing. The smaller number of counts corresponds to the smaller earth-satellite earth- sun angle. The larger the angle $\beta$ (called in this paper the sun-orbit angle) between the sun and the orbital plane, the deeper the measurements made in the hydrogen geocorona. This is why orbits in such configurations show a smaller number of counts. In the first analysis of data, orbits having a $\beta$ angle smaller than $10^{\circ}$ were considered. This was done to compare similar orbits in the earth's geocorona. All geocoronal results presented in this paper were obtained mainly by comparing data of the type shown in Figure 3 with an exospheric model combined with a model of the center of the solar Lyman $\dot{\alpha}$ line.

\section{Data Analysis}

\section{Solar Flux in the 100-A Bandpass}

The optical transmissions of the two channels giving the solar flux in the 100-A bandpass are very sensitive to spacecraft attitude, to the spin rate $S$ of the wheel where this experiment is installed, and to the pitch angle $P$ (angle between the solar vector and the wheel plane).

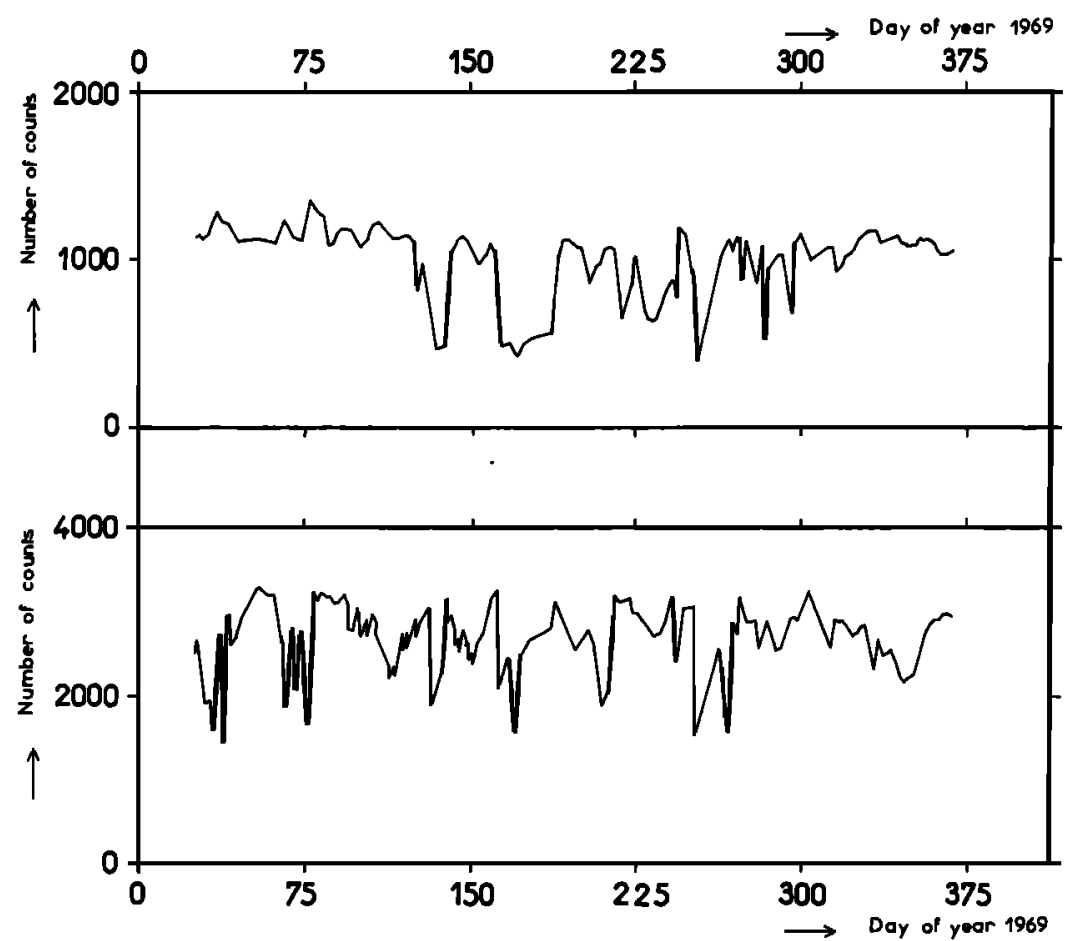

Fig. 2. Total solar Lyman $\alpha$ flux measured by the experiment through two independent channels: top, hydrogen channel; bottom, deuterium channel. 


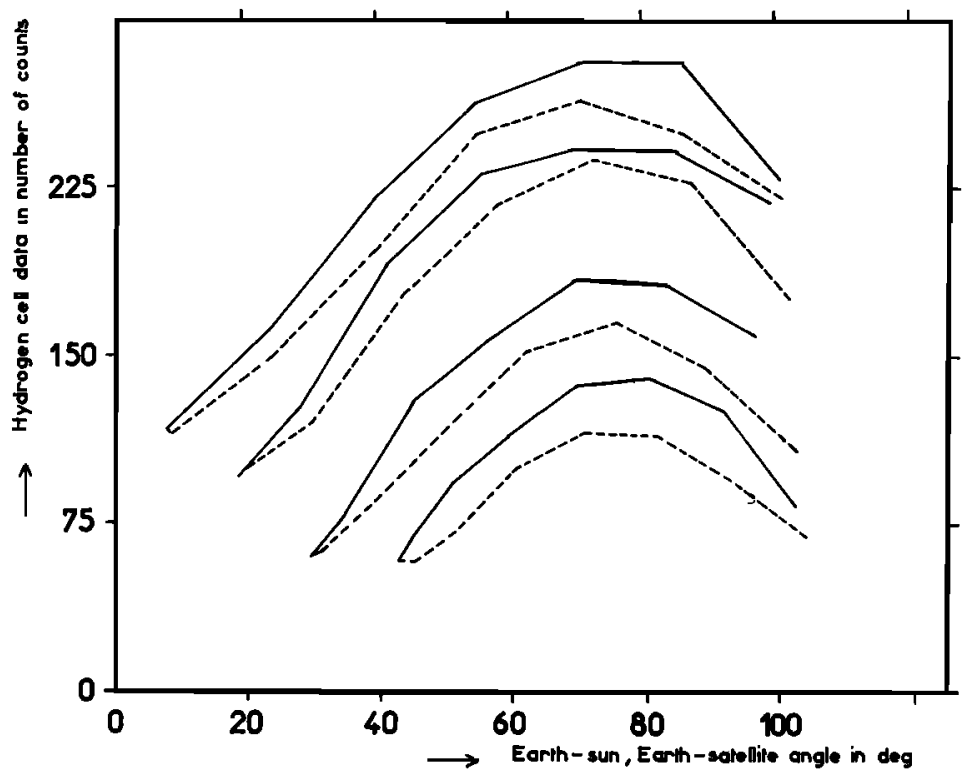

Fig. 3. Flux at the center of the solar Lyman $\alpha$ line as measured by the hydrogen cell : solid line, blue wing; dashed line, red wing.

For each pair of $S$ and $P$ values, a different transmission value exists. If $T_{1}(S, P)$ and $T_{2}(S, P)$ are the two transmission patterns of the two different channels, we can write

$$
\begin{aligned}
& F_{\text {. }}=M_{1} / T_{1}(S, P) \\
& F_{\text {. }}=M_{2} / T_{2}(S, P)
\end{aligned}
$$

where $M_{1}$ and $M_{2}$ are the data obtained in each one of these two channels and $F$, is the solar flux in the 100-A bandpass integrated over the whole solar disk. Obviously, these two equations are not sufficient to provide the solar flux if the transmission patterns $T_{1}$ and $T_{\mathrm{z}}$ are not known. $T_{1}$ and $T_{2}$ were measured before launch during the calibration of the experiment. This calibration procedure was quite complicated, and the two transmission patterns obtained were only rough approximations of the real transmission patterns of the two channels. Thus the solar flux measurements obtained through these two channels were still sensitive to spacecraft attitude after the two calibrated transmission patterns were applied to the data. These patterns then had to be modified in order to obtain a coherent value for the solar flux measured by the two channels. The technique used was largely manual: starting from regions of the pattern where the transmission revealed less sensitivity to spacecraft attitude, it was possible to modify the pattern step by step out of this region, keeping similar and smooth variations of the solar flux from both channels.

This was done until all the rapid variations shown in Figure 2 were erased by modifying the original transmission patterns, yet maintaining the smooth variations in these patterns on both axes. At the end of this process, the two patterns were within $\pm 20 \%$ of the original ones, giving two variations of the solar flux $F$. that agreed with each other, the average values of which are shown in Figure 4. The quality of this determination of the solar flux can then be evaluated by examining the two solar eclipses observed by the spacecraft on March 7 and August 31, 1970. As the point of measurement taken just before each eclipse was very close to that taken just after, the relative changes observed seem to be real.

An absolute calibration was made 3 months before launch for each channel over the whole transmission pattern (over the entire field of view: $8^{\circ}$ by $8^{\circ}$ ). This measurement was more precise than measurements of the shape of the pattern, because the latter were done with a $30^{\circ}$ by $30^{\prime}$ field of view, so that there were fewer counts in the detector. As it is not affected 
by the $\pm 20 \%$ change of the pattern, the error in the absolute calibration of the two channels is mainly due to the quality of the standard used. The standard, a $\mathbf{C S}_{2}$ ionization chamber, was calibrated in our laboratory by comparison with a flowing NO ionization chamber. It was then recalibrated, by the same technique, in the Laboratory for Atmospheric and Space Physies, Boulder, Colorado, yielding the same value within $\pm 3 \%$. As the quality of an absolute calibration is very hard to estimate, all the results presented here are given, for all channels, according to the last calibration made with our standard 3 months before launch. Although the quality of such a calibration may be good only during a short period after launch, the experiment seems to age very slowly, as it operates during only 1 hour every 2 calendar days. In fact, no important decay of the data appeared even after 2 years of operation (see Figure 4 ).

Solar Flux in a 0.01-A Bandpass at the Center of the line

The apparatus function of the hydrogen resonance cell is, as defined by Blamont and Vidal-Madjar [1971],

$$
\begin{gathered}
R(N)=\frac{K \Omega}{4 \pi} A(N) \int_{0}^{\infty} I(\lambda)\left[\exp \left(-N k_{\lambda} a\right)\right. \\
\left.-\exp \left(-N k_{\lambda} b\right)\right] d \lambda
\end{gathered}
$$

where $A(N)$ represents the absorption of the reemitted resonance light in the resonance branch:

$$
\begin{aligned}
& A(N)=\frac{1}{(\pi)^{1 / 2}} \frac{1}{\Delta \lambda_{D}} \\
& \quad \int_{0}^{\infty} \exp \left[-\left(\Delta \lambda / \Delta \lambda_{D}\right)^{2}-N k_{\lambda} d\right] d \lambda
\end{aligned}
$$

(see Blamont and Vidal-Madjar [1971] for the definition of all parameters). The resonance signal obtained, $R(N)$, is a function of $N$, the number of atoms in the cell per cubic centimeter. $K$ is a constant representing the effciency of the cell. The experiment was operated so that the same $I(\lambda)$ profile was observed through the cell for three different values of $N$. Starting from three calibrated values of the cell density, it was then possible to evaluate the aging of the cell during the satellite's life in the following way: if the three densities $N_{1}, N_{2}$,

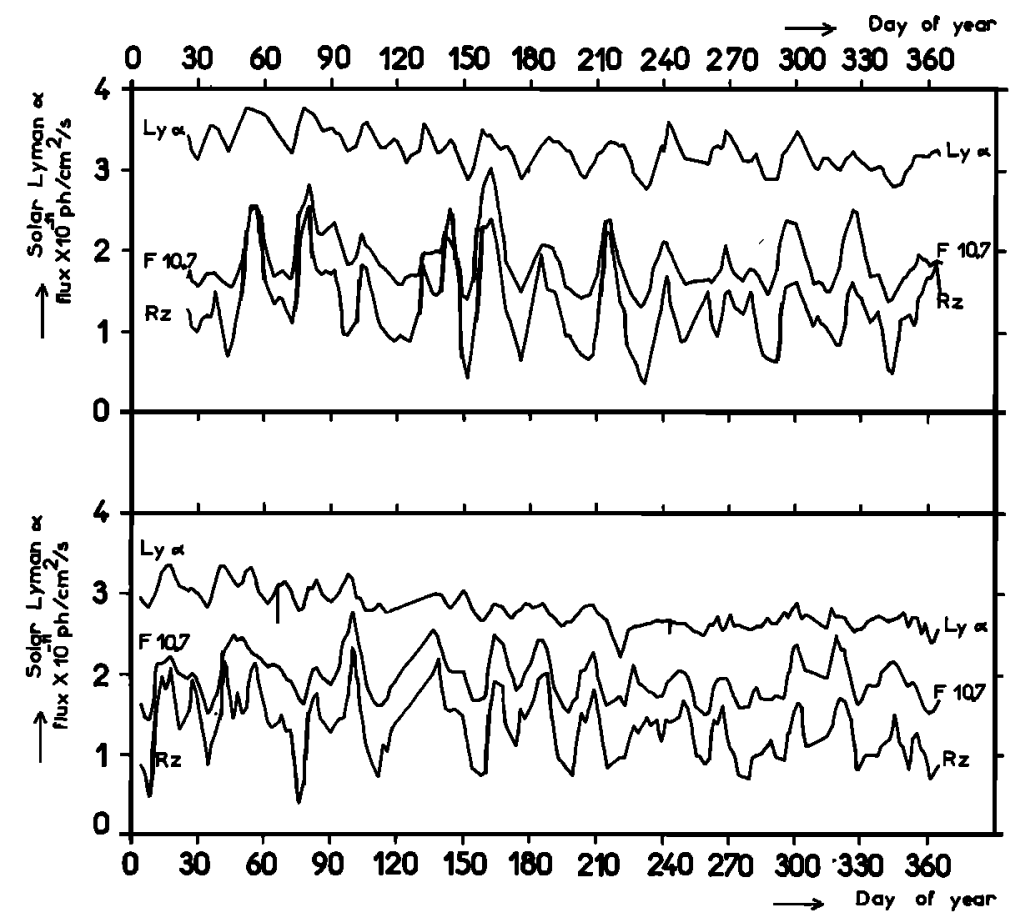

Fig. 4. The total solar Lyman $\alpha$ flux variations during 1969 (top) and 1970 (bottom). 
and $N_{3}$ corresponding to the three operational modes of the cell are known, as is the case for the first orbit, we are able to find for a given orbit the five best parameters (three solar and two geocoronal, as will be explained in the next paragraph) defining the function $I(\lambda)$ by use of the nonlinear least squares method discussed below. If $N_{1}, N_{2}$, and $N_{3}$ are not known, we obtain the best possible fit of $C$ as:

$$
\begin{aligned}
C\left(N_{1}, N_{2}, N_{3}\right) & =\sum\left(R\left(N_{1}\right)-M_{1}\right)^{2} \\
& +\sum\left(R\left(N_{2}\right)-M_{2}\right)^{2} \\
& +\sum\left(R\left(N_{3}\right)-M_{3}\right)^{2}
\end{aligned}
$$

where $M_{1}, M_{2}$, and $M_{3}$ represent the measurements made with the cell in the operational mode corresponding to $N_{1}, N_{2}$, and $N_{\mathrm{s}}$, respectively. The sums are over all the measured points. By finding the set $\left(N_{1}, N_{2}, N_{3}\right)$ that gives a minimum value for the function $C$, we obtained the three best values for the densities in the cell. Because from one orbit of measurement to the next the values $\left(N_{1}, N_{\mathrm{g}}, N_{\mathrm{g}}\right)$ change very slowly, it was possible to trace with ease the minimum of the function $C$ along the successive orbits of measurement. This survey of the cell was made over 2 years and demonstrates the rate of aging (see Figure 5). These values of $\left(N_{1}, N_{2}, N_{\mathrm{s}}\right)$ defining the hydrogen resonance cell were then used during the data processing.

\section{Different Exospheric Models Used for the} Interpretation of the Resonance Hydrogen Data

Chamberlain exospheric model. The data obtained through the hydrogen resonance channel as described by Blamont and Vidal-Madjar [1971] are used in the comparison. These authors explain particularly how the data can be compared to a five-parameter model: three parameters are used for the parabolic shape of the solar central reversal, and two are used to represent the geocoronal hydrogen absorption (temperature and optical thickness). We tried here to use a more sophisticated model of the geocoronal hydrogen absorption based on the Chamberlain [1963] exospheric model, which is a density model having spherical symmetry and defined by only two parameters: the temperature and density at the exobase level (the exobase is the base of the exosphere, taken here to be at an altitude of $500 \mathrm{~km}$ ). It was possible to calculate, along a given line of sight, the integral of atoms having a given projection of their velocity. This calculation, made for all possible projected velocities, gave the absorption line profile at that specific point of the exosphere along that particular line of sight.

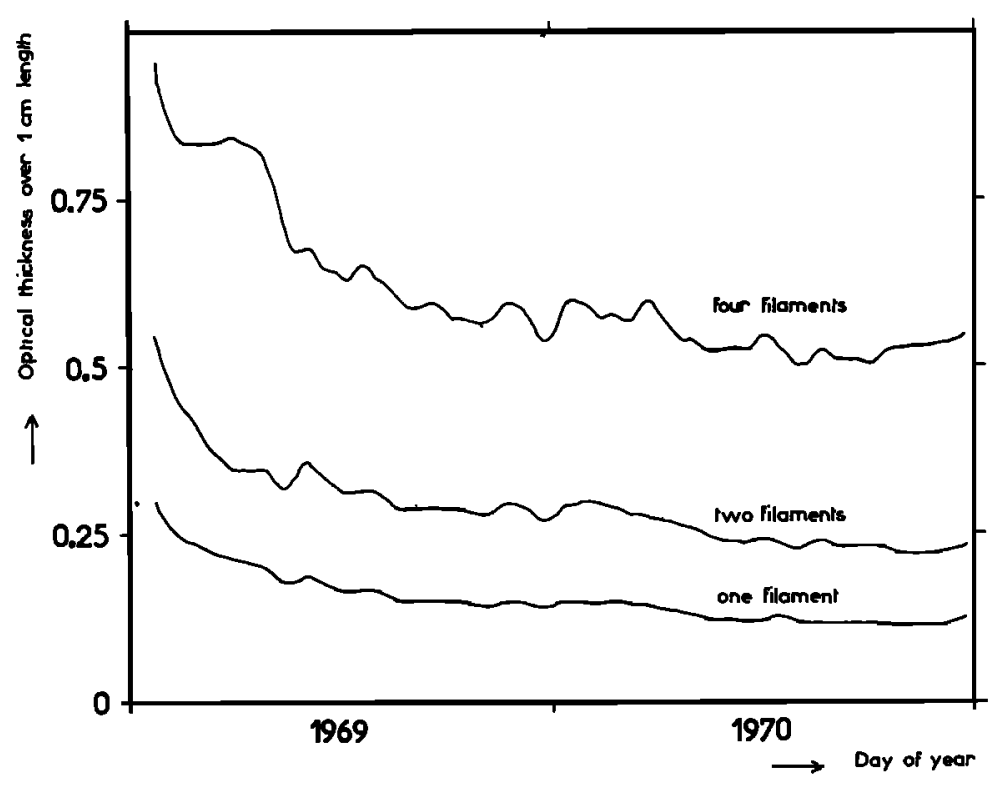

Fig. 5. Aging of the hydrogen resonance cell. 
Because the orbit is circular at an altitude of $550 \mathrm{~km}$, and the model taken is spherically symmetrical, it was then enough to calculate the absorption profile for a given model (density and temperature chosen at the exobase) and at an altitude of $550 \mathrm{~km}$ for all possible zenith angles defining the line of sight. As density is a multiplicative factor in the Chamberlain exospheric model, all possible absorption line profiles to be observed from the 550$\mathrm{km}$ circular orbit could be represented solely by a change in temperature. This calculation was made by J. L. Bertaux and J. C. Lebrun (private communication, 1972) for different steps of each variable:

Projection of the velocity in steps of $0.005 \mathrm{~A}$.

Zenith angle in steps of $10^{\circ}$.

Exospheric temperature in steps of $250^{\circ} \mathrm{K}$.

They showed that the addition of satellite particles as defined by Chamberlain [1963], with a critical satellite level of $2.5 R_{E}$, changed the calculated absorption profiles less than 1\%. For this reason, the Chamberlain model used here does not take satellite particles into account. Thus it is possible to find by linear interpolation the best temperature fitting the data.

Comparison of the data to a five-parameter model. In the five-parameter model, three solar parameters approximate the solar Lyman $\alpha$ reversal by a parabolic shape:

$I_{0}(\lambda)=a\left(\lambda-\lambda_{0}\right)^{2}+b\left(\lambda-\lambda_{0}\right)+c$

where $a$ is the concavity parameter, $b$ is the asymmetry parameter, and $c$ is the central flux of the solar line. To evaluate $c$ in an absolute sense the experiment's calibration before launch was used. The hydrogen resonance cell was at that time calibrated by the method described by Blamont and Vidal-Madjar [1971]; because of the difficulty of this type of calibration, results have an uncertainty of $\pm 20 \%$.

The two geocoronal parameters are atomic hydrogen density at the exobase (500 $\mathrm{km}$ ), and (2) exospheric temperature. The geocoronal model is the Chamberlain one having spherical symmetry.

These five parameters were introduced in (1), defining the line shape $I(\lambda)$ at the entrance of the cell:

$$
I(\lambda)=I_{0}(\lambda) \cdot \exp \left[-N F\left(T, \lambda-\lambda_{0}, \omega\right)\right]
$$

where $I_{0}(\lambda)$ is the solar reversal defined by (4), $N$ is the atomic hydrogen density at the exobase, and $T$ is the exospheric temperature. The function $F\left(T, \lambda-\lambda_{0}, \omega\right)$ represents the absorption in the geocorona at a point at $550-\mathrm{km}$ altitude (this function is the optical depth along a line of sight from the Chamberlain model, normalized to unit hydrogen density at $500 \mathrm{~km}$ ) in a direction having a given zenith angle $\omega$ at a given wavelength $\left(\lambda-\lambda_{0}\right)$, and for a given exospheric temperature. This function was calculated for different exospheric temperatures, the values $\omega$ and $\left(\lambda-\lambda_{0}\right)$ being known for each measurement. It is then possible to find by linear interpolation the best temperature that fits the data, along with the other four parameters of the least squares fit. As the function $F$ is not very sensitive to temperature, variation steps of $250^{\circ} \mathrm{K}$ were used for the linear interpolation.

It is important to note that in (2) a change in the $K$ coefficient will induce directly proportional changes in the $a, b, c$ parameters, which are functions of the experiment's absolute calibration. On the other hand, the coefficients $N$ and $T$ are independent of that absolute calibration, and their values will have then an absolute signification.

Description of the aspherical models. As we just saw, it is possible to compare the data to a five-parameter model composed of three solar parameters plus the two geocoronal parameters that represent the exobase hydrogen density and the exospheric temperature in Chamberlain's [1963] spherical exospheric model. Because systematic differences appeared between the measurements and the spherical model, several asymmetric exospheric models were constructed.

To understand how these aspherical exospheric models were constructed, one must remember that the exosphere observed in absorption from the exobase is mainly the lower exosphere (below $1 R_{E}$ in altitude). As a matter of fact, the integral of atoms along a line of sight (from $550 \mathrm{~km}$ to infinity) can be represented quite well by the integral of atoms intercepted up to $1 R_{u}$ in altitude. In Figure 6 these two integrals are compared for two particular lines of sight, showing that about $85 \%$ of the atoms absorbing the solar flux, as observed from 
an altitude of $550 \mathrm{~km}$, are below $1 R_{E}$ in altitude.

On the other hand, Vidal-Madjar and Bertaux [1972] showed that below $1 R_{B}$ altitude the exospheric densities are quite well approximated by what they call the local Chamberlain model $( \pm 10 \%)$, which is, in fact, a spherical Chamberlain exospheric model whose parameters are the local density and temperature. This means that for each point of the exobase a different Chamberlain exospheric model is used.

Therefore all aspherical exospheric models used are constructed according to the following method: A density and temperature gradient is defined at the exobase. The absorption along any line of sight is then evaluated by assuming that this line of sight is immersed in a spherical Chamberlain exospheric model whose parameters are the temperature and density at the point of the exobase just below the origin of the line of sight.

Under these conditions, all the calculations presented in the previous paragraphs are still valid, and, particularly for all the aspherical models, will still be used. In the aspherical models we use a relation that is very similar to (5). The line shape $I(\lambda)$ at the entrance of the cell is now

$$
\begin{aligned}
& I(\lambda)=I_{0}(\lambda) \\
& \cdot \exp \left\{-N(\alpha, \delta) F\left[T(\alpha, \delta), \lambda-\lambda_{0}, \omega\right]\right\}
\end{aligned}
$$

where $N(\alpha, \delta)$ and $T(\alpha, \delta)$ are two functions of local time $\alpha$ and latitude $\delta$ representing the variations of the hydrogen density and of the exospheric temperature at the exobase.

Different aspherical exospheric models. In the Jacchia, Kockarts and Nicolet model we assume that temperature and density are known. For the temperature we use the Jacchia [1971] model giving the $T(\alpha, \delta)$ distribution, and for the density we use the Jacchia [1971] approximation of the Kockarts and Nicolet [1963] hydrogen density distribution at the exobase, giving the $N(\alpha, \delta)$ function.

In the Jacchia nonspherical model only temperatures are assumed to be known, and again the $T(\alpha, \delta)$ function represents the Jacchia [1971] exospheric temperature distribution. To define the density $N(\alpha, \delta)$ a simple relation is constructed in the following way: If we assume

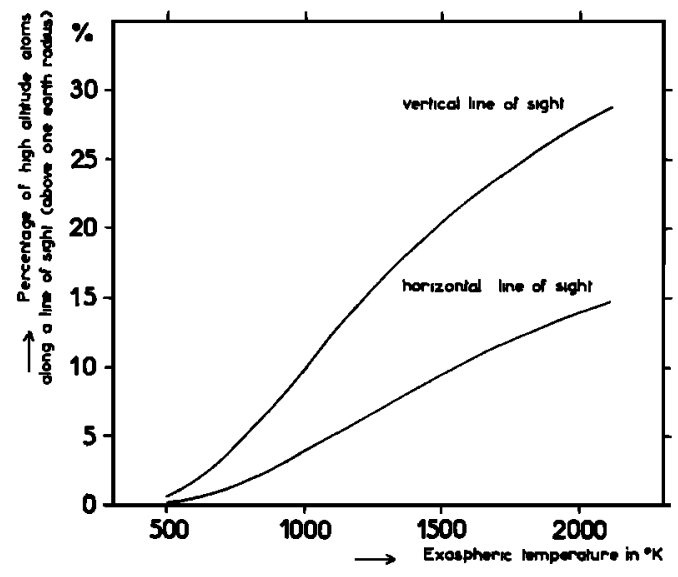

Fig. 6. Percentage of high-altitude atoms (above $1 R_{E}$ ) along particular lines of sight starting at an altitude of $550 \mathrm{~km}$.

that a linear relation exists between $T$ and $N$ and that the minimum and maximum density $H_{\min }$ and $H_{\max }$ correspond, respectively, to the maximum and minimum temperatures $T_{\max }$ and $T_{\mathrm{m} 1 \mathrm{n}}$, we can write:

$$
\begin{aligned}
N(\alpha, \delta)= & \frac{H_{\max }-H_{\min }}{T_{\max }-T_{\min }} T(\alpha, \delta) \\
& +\frac{H_{\max } T_{\max }-H_{\min } T_{\min }}{T_{\max }-T_{\min }}
\end{aligned}
$$

In such a model, where the temperatures are known, two geocoronal parameters appear: $H_{\min }$ and $H_{\max }$, the minimum and maximum hydrogen density at the exobase.

For the axial symmetry model, since our measurements are not very sensitive to temperature and because measurements are made only on the day side of the earth, it seems reasonable to use sinusoidal representations of the temperature and density variations. The Jacchia [1971] model is then used only to obtain minimum and maximum values of the exospheric temperature at the moment of a given orbit of measurement. Such a model will have a symmetry axis defined by $\left(\alpha_{\mathrm{nym}}, \delta_{\mathrm{aym}}\right)$, the direction of the point of maximum temperature in local time and declination. If a particular point of the exobase is defined by $(\alpha, \delta)$ we can then write 
$T(\alpha, \delta)=\frac{T_{\operatorname{mix}}+T_{\min }}{2}$

$$
+\frac{T_{\max }-T_{\min }}{2} \cos \gamma
$$

$$
\begin{aligned}
N(\alpha, \delta)=\frac{H_{\max }+H_{\min }}{2} & \\
& -\frac{H_{\max }-H_{\min }}{2} \cos \gamma
\end{aligned}
$$

where $\gamma$ is the angle between the directions $(\alpha, \delta)$ and $\left(\alpha_{\text {oym }}, \delta_{\text {oym }}\right)$. We assume again here that the maximum and minimum temperatures correspond to the minimum and maximum densities, respectively.

In such an exospheric model, the geocoronal parameters are now as follows: (1) If $T_{\min }$ is assumed to be known and equal to $T_{\text {。 }}$ (Jacchia 1971 model) we can introduce the ratio $T_{\max } /$ $T_{\min }$ as an unknown value. (2) The direction of the exobase symmetry axis $\left(\alpha_{\mathrm{sym}}, \delta_{\mathrm{sym}}\right)$ may be changed. (3) The two important geocoronal parameters are, in fact, the minimum and maximum densities $H_{\mathrm{m} \text { ln }}$ and $H_{\mathrm{max}}$.

Nevertheless, these different parameters are treated in different ways: the ratio $T_{\min } / T_{\max }$ and the values $\alpha_{\text {aym }}$ and $\delta_{\text {oym }}$ are not critical. This is why in all the calculations they are treated as constant values. Only the systematic differences appearing between models and measurements are used to decide which one is the best:

The following values are tried for $T_{\max } / T_{\min }$ : 1.1, 1.3, 1.5 .

The following values are tried for $\alpha_{8 y \mathrm{~m}}: 1200$, $1400,1600,1800$ local time.

The value for $\delta_{, y m}$ is always taken to be the solar declination.

Large steps are used because of the insensitivity of these parameters. The last two parameters $H_{\min }$ and $H_{\max }$ are then found through the nonlinear least squares fit, as was explained.

\section{Results}

Monitoring of the Solar Lyman a Flux during the Years 1969 and 1970

As is shown in Figure 4, the total solar Lyman $\alpha$ flux is plotted as a function of the day during the years 1969 and 1970, roughly one measure- ment point being taken every two days. The maximum flux variation observed during one solar rotation is of the order of $30 \%$, and the correlations of all variations with the Zurich sunspot number or with the $10.7-\mathrm{cm}$ solar flux are quite good, as is shown in Figure 7.

Another experiment on the Nimbus 3 and 4 spacecraft measured the total solar Lyman $\alpha$ flux in 1969 and 1970 (D. G. Heath, unpublished manuscript, 1971). The correlation with these data is excellent.

According to our calibration the average value of the solar Lyman $\alpha$ flux is of the order of $3 \times 10^{11} \mathrm{ph} \mathrm{cm}^{-2} \mathrm{sec}^{-1}$. In Table 1, the results are presented for all measurement orbits during 1969 and 1970. In 1970, two solar eclipses were observed, on days 66.68 and 243.84-243.89. The decrease of the Lyman $\alpha$ flux measured during these three orbits is solely related to the geometry of each orbit passing through the moon's shadow.

A later section contains a correlation coefficient of 0.87 between this total Lyman a solar flux and a completely independent measurement of the solar flux at the center of the line, showing the high degree of confidence that one can have in the values given in Table 1.

\section{Results Obtained with the Resonance Hydrogen Data}

Comparison with a five-parameter spherical model. The five-parameter model is divided into two parts. For the part concerned with the solar central line shape, the center of the line is assumed to be parabolic (see equation 4), and three parameters are used: $a$ is the concavity of the solar reversal; $b$ is the central asymmetry, and $c$ is the solar flux at the center of the line. For the geocoronal part of the model, the Chamberlain exospheric model defined by two parameters is used: $N_{H}$ is the density of atomic hydrogen at the exobase $(500 \mathrm{~km})$, and $T$ is the exospheric temperature.

Comparison with the five-parameter model is made only with orbits whose planes are within $10^{\circ}$ of the direction of the sun. Figure 8 shows how the five parameters vary with solar activity. The solar activity is estimated here by the Zurich sunspot number.

The first important result found was the existence of a correlation between some parameters and solar activity. The best correlations 


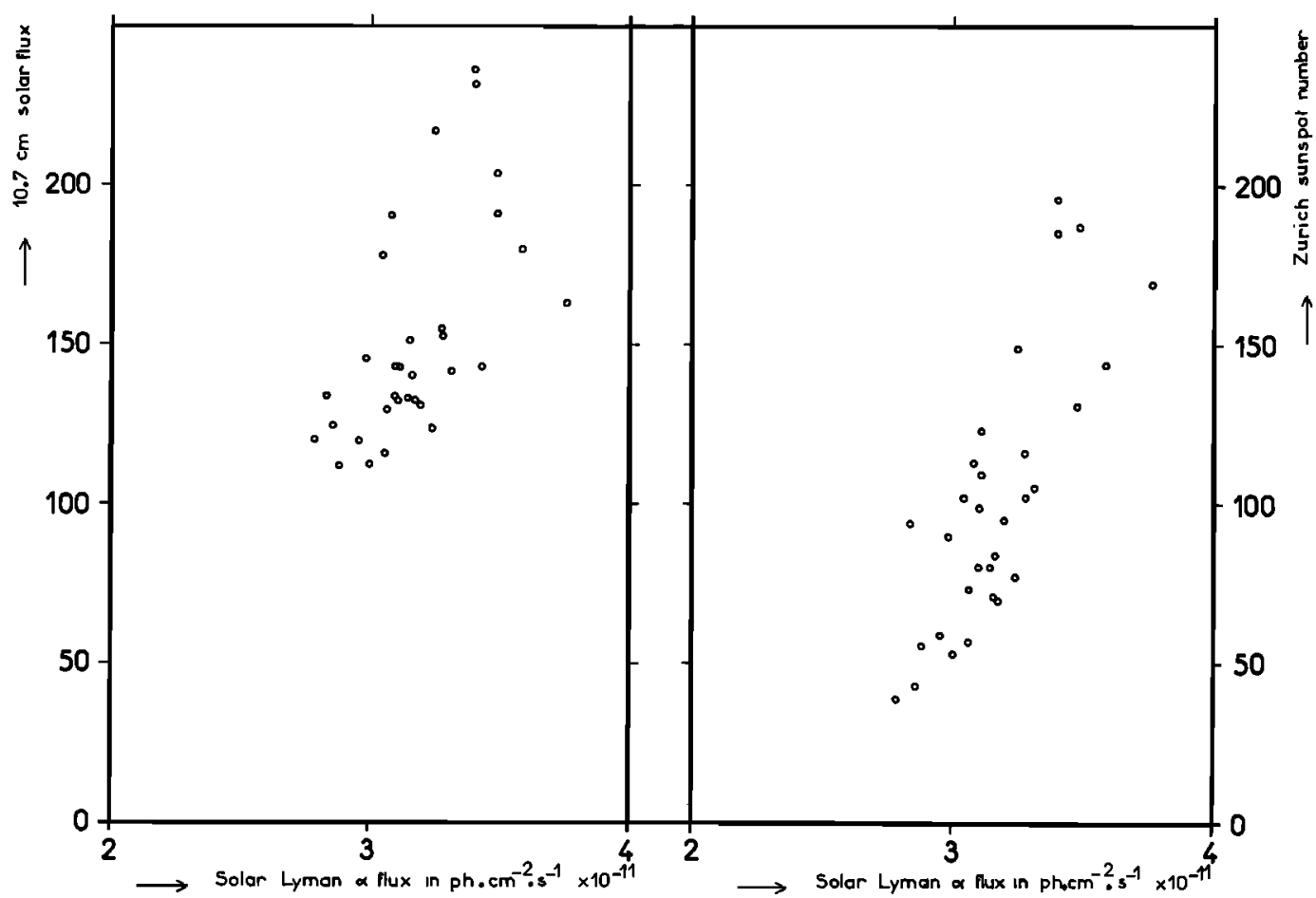

Fig. 7. Correlation of the total Lyman $\alpha$ flux with the Zurich sunspot number and with the $10.7-\mathrm{cm}$ solar flux.

observed were for the solar symmetry parameter $c$ (showing a systematic stronger emission in the blue wing than in the red wing of the line) and for the atomic hydrogen density at $500 \mathrm{~km}$ (behaving as expected, density decreasing as solar activity increased).

Nevertheless, the very large error bars found for the solar concavity parameter and for the exospheric temperature value suggest that the accuracy of the measurement is not sufficient to determine these two parameters.

As a matter of fact, these two parameters have equivalent roles in the model and we can say that both of them are concavity parameters. This is why a quasi-linear relation is found between these two parameters (Figure 9), showing that the meaning of the solar concavity parameter is very doubtful. The fact that this parameter always presents the central solar reversal as an emission feature may be an indication but no more. Therefore we suppress one of the two concavity parameters and assume henceforth that the bottom of the solar reversal is flat $(a=0)$, since we do not have enough information to make another assumption and since this assumption seems to change nothing in our analysis.

Comparison with a four-parameter spherical model. The same model was used with a flat bottom of the solar reversal. The parameters are those described for the five-parameter model, $a$ being always equal to zero.

Figure 10 presents the variations of the four other parameters with solar activity for orbits having a sun-orbit angle smaller than $10^{\circ}$. Correlations appear for all parameters but the exospheric temperature. The central solar slope (symmetry parameter $b$ ) and the central solar flux increase with solar activity, and again the exospheric density decreases with increasing solar activity. The fact that the exospheric temperature error bar remains very large shows definitely that this kind of measurement is not at all sensitive to exospheric temperature. On the other hand, the correlation between the other parameters and solar activity is still very poor (in particular, the correlation coefficient between the central solar flux and the Zurich 
TABLE 1. Total Solar Lyman $\alpha$ Flux $\times 10^{\text {u }}$

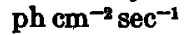

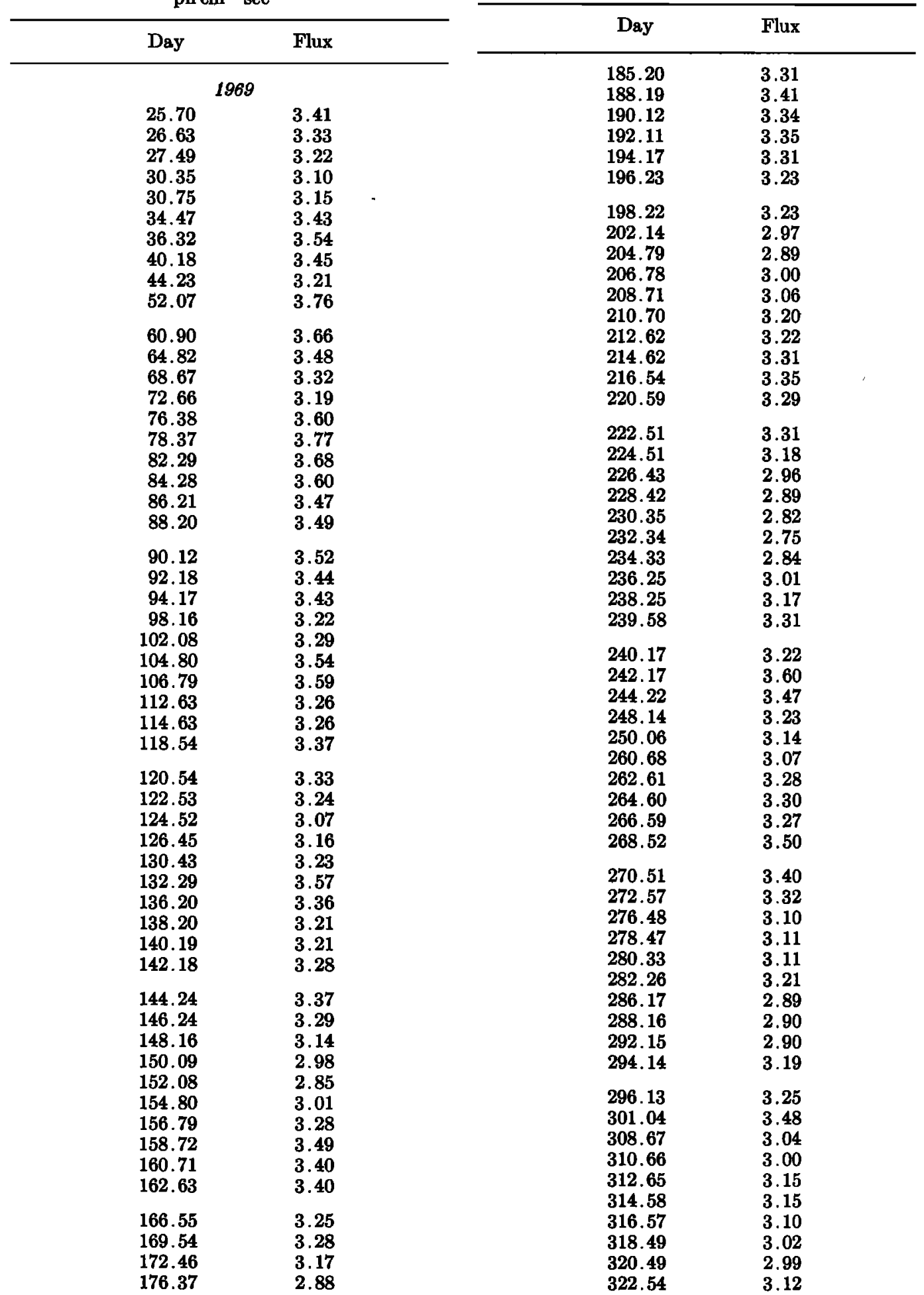

TABLE 1. (continued) 
Vidal-Madjar et al.: Solar Lyman $\alpha$ and Hydrogen Exobase

TABLE 1. (continued)

\begin{tabular}{|c|c|c|c|}
\hline Day & Flux & Day & Flux \\
\hline 324.53 & 3.18 & 78.10 & 2.78 \\
\hline 326.39 & 3.24 & 80.16 & 3.06 \\
\hline 328.32 & 3.16 & 82.09 & 3.02 \\
\hline 330.31 & 3.08 & 84.07 & 3.14 \\
\hline 332.23 & 3.05 & 86.06 & 2.99 \\
\hline 334.22 & 2.99 & 87.99 & 2.91 \\
\hline 338.14 & 3.05 & & \\
\hline 340.13 & 3.00 & 90.71 & 2.86 \\
\hline 342.12 & 2.86 & 94.69 & 2.99 \\
\hline 344.11 & 2.79 & 96.61 & 3.10 \\
\hline 348.09 & & 98.60 & 3.22 \\
\hline $\begin{array}{l}348.09 \\
350.08\end{array}$ & $\begin{array}{l}2.83 \\
2.98\end{array}$ & 100.60 & 3.16 \\
\hline $\begin{array}{l}350.08 \\
352.00\end{array}$ & & 100.66 & 3.14 \\
\hline & $\begin{array}{l}3.05 \\
3.18\end{array}$ & 102.52 & 2.91 \\
\hline $\begin{array}{r}353.99 \\
355.72\end{array}$ & 3.18 & 104.50 & 2.90 \\
\hline 355.72 & 3.18 & 106.49 & 2.74 \\
\hline $\begin{array}{l}357.71 \\
359.70\end{array}$ & $\begin{array}{l}3.17 \\
3.16\end{array}$ & 110.34 & 2.78 \\
\hline 361.63 & $\begin{array}{l}3.10 \\
3.23\end{array}$ & 112.33 & 2.85 \\
\hline 363.62 & $\begin{array}{l}0.20 \\
3.26\end{array}$ & $\begin{array}{l}112.35 \\
114.32\end{array}$ & $\begin{array}{l}2.80 \\
2.78\end{array}$ \\
\hline 365.54 & 3.20 & 116.31 & 2.71 \\
\hline \multirow{2}{*}{\multicolumn{2}{|c|}{1970}} & 118.30 & 2.77 \\
\hline & & 136.81 & 2.96 \\
\hline 4.59 & 2.94 & 139.06 & 2.96 \\
\hline 6.45 & 2.85 & 140.72 & 2.94 \\
\hline 8.44 & 2.79 & 142.77 & 2.84 \\
\hline 10.37 & 2.91 & 144.70 & 2.78 \\
\hline 12.36 & 3.06 & 146.62 & 2.84 \\
\hline 14.28 & 3.25 & 150.53 & 3.00 \\
\hline 16.27 & 3.32 & 154.45 & 2.77 \\
\hline 18.26 & 3.33 & $\begin{array}{l}158.45 \\
158.36\end{array}$ & 2.62 \\
\hline 20.18 & 3.18 & 160.35 & 2.64 \\
\hline 22.17 & 3.06 & 162.33 & 2.72 \\
\hline 26.16 & 3.00 & 164.26 & 2.83 \\
\hline 28.15 & 3.03 & 168.24 & 2.78 \\
\hline 30.14 & 2.98 & 170.16 & 2.70 \\
\hline 35.04 & 2.80 & 172.15 & 2.65 \\
\hline 37.03 & 2.95 & 174.21 & 2.74 \\
\hline 38.76 & 3.15 & & \\
\hline 40.75 & 3.31 & $\begin{array}{l}178.20 \\
178.19\end{array}$ & $\begin{array}{l}2.10 \\
2.83\end{array}$ \\
\hline 42.67 & 3.31 & 180.18 & 2.83 \\
\hline $\begin{array}{l}46.66 \\
48.58\end{array}$ & $\begin{array}{l}\text { 3.13 } \\
\text { 3.07 }\end{array}$ & 182.17 & 2.79 \\
\hline & & 184.02 & 2.82 \\
\hline 50.57 & 3.10 & 186.02 & 2.86 \\
\hline 52.50 & 3.25 & 188.00 & 2.75 \\
\hline 54.55 & 3.31 & 191.71 & 2.64 \\
\hline 56.48 & 3.18 & 193.64 & 2.58 \\
\hline 58.40 & 2.98 & 195.63 & 2.57 \\
\hline 62.38 & 2.84 & 197.62 & 2.62 \\
\hline 64.37 & 2.93 & $\begin{array}{l}197.62 \\
199.54\end{array}$ & 2.61 \\
\hline 66.63 & 3.08 & 201.53 & 2.70 \\
\hline $\begin{array}{l}66.68 \\
66.76\end{array}$ & $\begin{array}{r}2.60 \\
3.08\end{array}$ & 203.45 & 2.77 \\
\hline 66.76 & 3.08 & 205.44 & 2.80 \\
\hline 68.29 & 3.08 & 209.35 & 2.82 \\
\hline 70.28 & 3.12 & 211.34 & 2.71 \\
\hline 72.20 & 3.04 & 215.25 & 2.61 \\
\hline 74.19 & 2.89 & 221.22 & 2.16 \\
\hline 76.11 & 2.76 & 223.14 & 2.31 \\
\hline
\end{tabular}

TABLE 1. (continued) 
TABLE 1. (continued)

\begin{tabular}{|c|c|}
\hline Day & Flux \\
\hline $\begin{array}{l}225.13 \\
227.19 \\
229.11 \\
233.09 \\
235.01 \\
237.80 \\
239.79 \\
241.77 \\
243.76 \\
243.84\end{array}$ & $\begin{array}{l}2.52 \\
2.55 \\
2.55 \\
2.59 \\
2.59 \\
2.63 \\
2.61 \\
2.62 \\
2.63 \\
2.45\end{array}$ \\
\hline $\begin{array}{l}243.89 \\
243.96 \\
247.61 \\
249.60 \\
251.52 \\
253.45 \\
255.43 \\
257.42 \\
259.35 \\
261.33\end{array}$ & $\begin{array}{l}2.54 \\
2.62 \\
2.58 \\
2.59 \\
2.56 \\
2.51 \\
2.48 \\
2.45 \\
2.43 \\
2.58\end{array}$ \\
\hline $\begin{array}{l}263.33 \\
265.31 \\
267.30 \\
269.22 \\
271.21 \\
273.14 \\
275.19 \\
279.17 \\
281.16 \\
283.15\end{array}$ & $\begin{array}{l}2.57 \\
2.69 \\
2.49 \\
2.55 \\
2.72 \\
2.55 \\
2.57 \\
2.52 \\
2.52 \\
2.56\end{array}$ \\
\hline $\begin{array}{l}285.07 \\
287.92 \\
289.71 \\
291.70 \\
295.61 \\
297.07 \\
297.27 \\
297.53 \\
297.73 \\
297.86\end{array}$ & $\begin{array}{l}2.55 \\
2.67 \\
2.60 \\
2.58 \\
2.74 \\
2.70 \\
2.66 \\
2.66 \\
2.66 \\
2.67\end{array}$ \\
\hline $\begin{array}{l}299.52 \\
301.58 \\
303.50 \\
305.49 \\
315.23 \\
317.29 \\
319.21 \\
321.20 \\
323.12 \\
325.11\end{array}$ & $\begin{array}{l}2.77 \\
2.86 \\
2.65 \\
2.70 \\
2.52 \\
2.79 \\
2.66 \\
2.66 \\
2.64 \\
2.55\end{array}$ \\
\hline $\begin{array}{l}327.10 \\
329.09 \\
331.14 \\
333.06 \\
337.97 \\
339.69 \\
341.68\end{array}$ & $\begin{array}{l}2.49 \\
2.51 \\
2.52 \\
2.60 \\
2.68 \\
2.67 \\
2.64\end{array}$ \\
\hline
\end{tabular}

TABLE 1. (continued)

\begin{tabular}{ll}
\hline Day & Flux \\
\hline 343.47 & 2.62 \\
343.60 & 2.62 \\
345.59 & 2.65 \\
347.58 & 2.66 \\
349.57 & 2.74 \\
351.56 & 2.54 \\
353.55 & 2.67 \\
355.40 & 2.66 \\
357.39 & 2.44 \\
359.31 & 2.60 \\
361.30 & 2.34 \\
363.29 & 2.37 \\
365.21 & 2.52 \\
\hline
\end{tabular}

sunspot number is equal to 0.38 ). This is why we tried to compare the variation of these parameters directly with the total solar Lyman $\alpha$ flux, as measured by that same instrument in a completely independent channel. It is surprising to see (Figure 11) how good these correlations are; they provide confidence simultaneously in the quality of this analysis and in the quality of the data processing leading to the total solar Lyman $\alpha$ flux, as was previously explained.

The correlation between the total solar flux and the central solar flux (correlation coefficient of 0.83 ) shows that the center line may change by a factor of $50 \%$ when the total line changes by only a factor of $30 \%$ (maximum variation observed during a solar rotation).

The correlation with the exospheric parameters and with the hydrogen density in particular is not as good. This is not surprising, since there is no physical reason to find perfectly correlated changes between the solar Lyman $\alpha$ flux and the hydrogen density at the exobase.

Finally, the correlation with the solar symmetry parameter (correlation coefficient equal to $\mathbf{0 . 4 8}$ ) is not very good either, since, as we will see in a later section, this parameter may represent geocoronal as well as solar asymmetries.

The main results obtained for the fourparameter model are as follows:

1. Correlation between the total solar Ly$\operatorname{man} \alpha$ flux and the center of the Lyman $\alpha$ solar flux is excellent ( 0.83 coefficient).

2. There is a $30 \%$ change in the total line flux corresponding to a $50 \%$ flux change at the center of the line. 

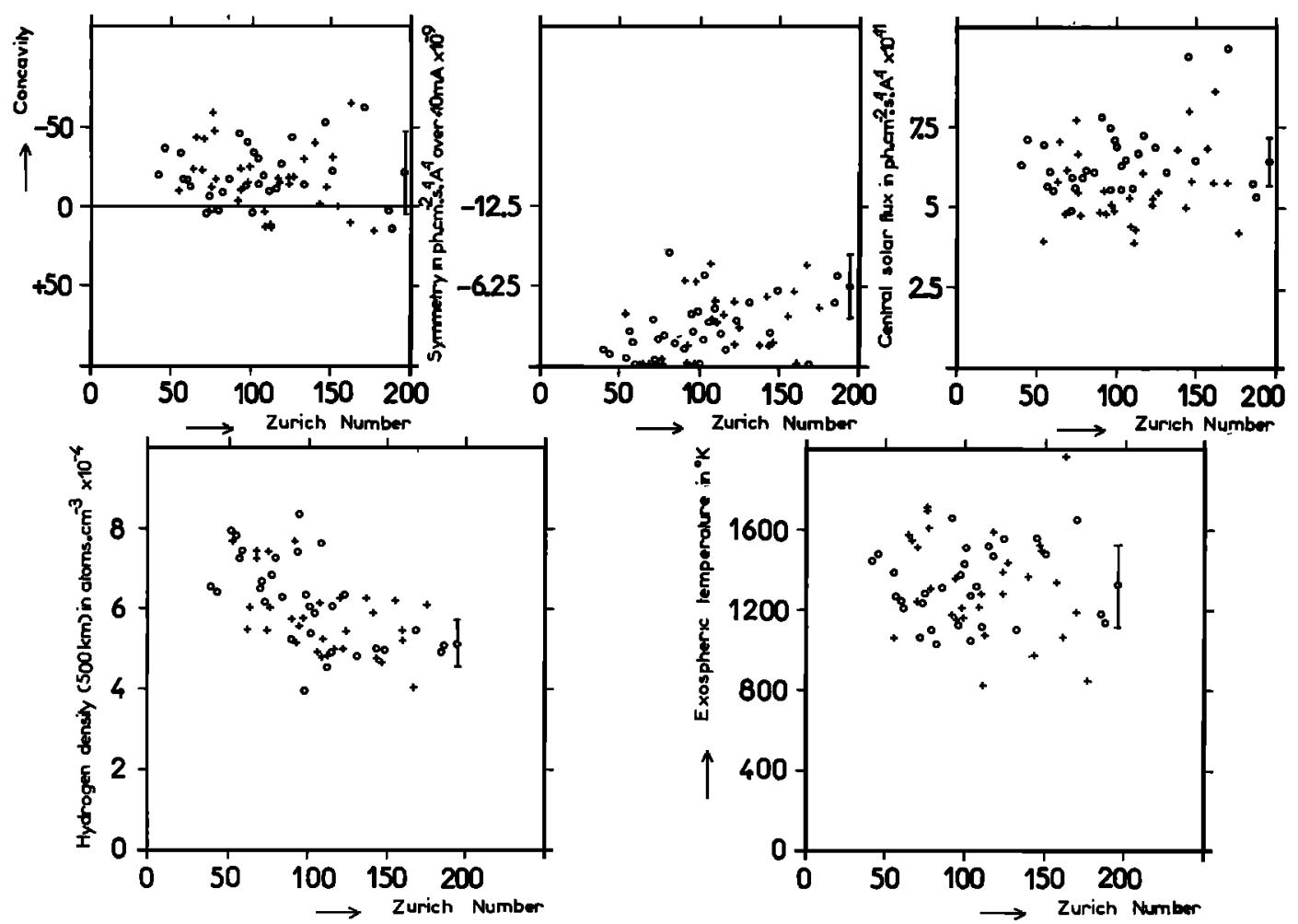

Fig. 8. Correlation of a five-parameter fit (three solar, two representing a spherical exosphere) with the Zurich sunspot number: circles, 1969; crosses, 1970.

3. The blue wing is more intense than the red wing.

4. According to our calibration the central solar flux is quite high, of the order of $5 \times 10^{11}$ ph $\mathrm{cm}^{-2} \sec ^{-1} \mathrm{~A}^{-1}$.

5. The average exospheric temperature on the day side is of the order of $1200^{\circ} \mathrm{K}$.

6. The hydrogen density at $500 \mathrm{~km}$ is measured in an absolute way (independent of the experiment's calibration) and is only a function of the geocoronal model used for comparison, varying from $4 \times 10^{4}$ to $8 \times 10^{4}$ atoms $\mathrm{cm}^{-8}$ As was expected, this hydrogen density decreases with increasing solar activity.

These differences between the measurement points and the model were evaluated in terms of wavelength, as each measurement is associated with a wavelength according to the Doppler shift (caused by the spacecraft's velocity, as was explained by Blamont and VidalMadjar [1971]). The differences were accumulated during a whole year in intervals of $2 \mathrm{~mA}$ over the whole scanning region from $\Delta \lambda=-30$ $\mathrm{mA}$ to $\Delta \lambda=+30 \mathrm{~mA}$. The average differences were then plotted as functions of wavelength (Figure 12).

The fact that the general shape found in

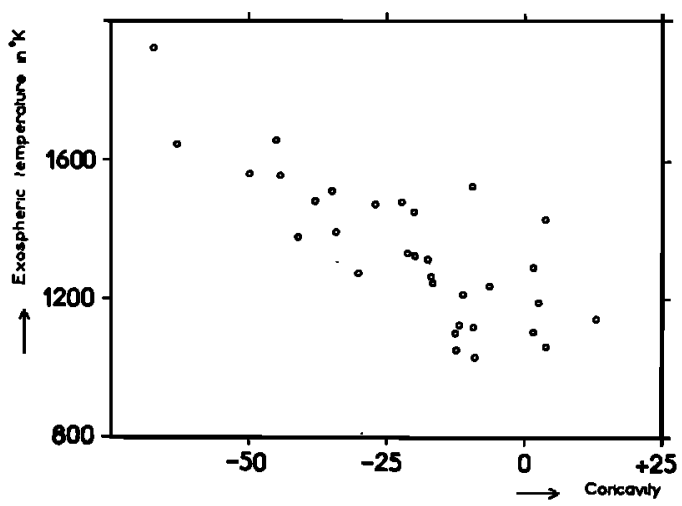

Fig. 9. Relation found between two parameters of the five-parameter fit (spherical exosphere, parabolic solar center) with 1969 data. 


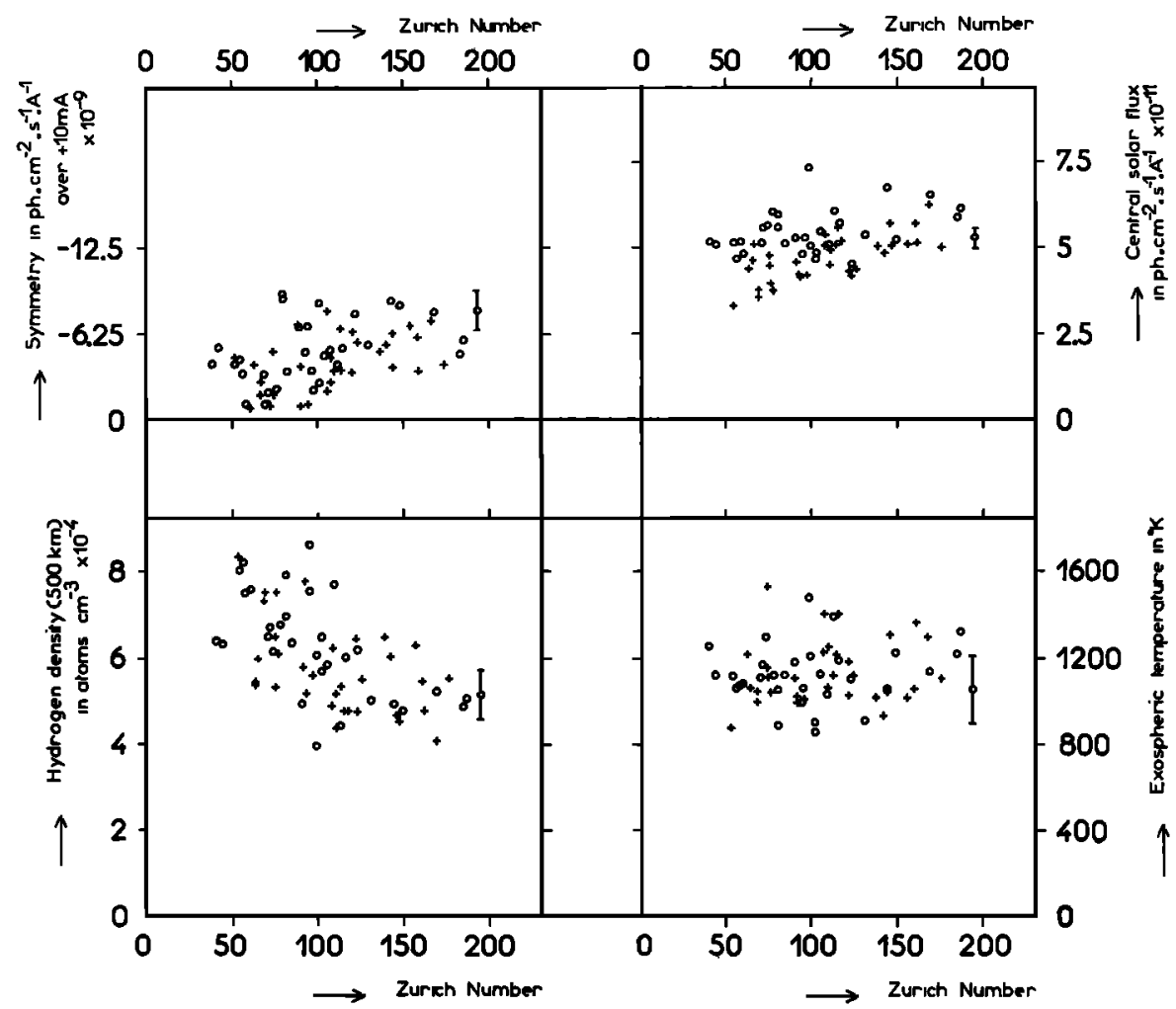

Fig. 10. Correlation of a four-parameter fit (flat solar center, spherical exosphere) with the Zurich sunspot number: circles, 1969; crosses, 1970.

1969 is found again in 1970 shows that these differences are real and proves that the spherical exospheric model is unrealistic. As a matter of fact, the model's evaluations are systematically above the measurements taken in the morning $(\Delta \lambda>0)$ and below those taken in the evening $(\Delta \lambda<0)$, showing that the geocoronal absorption is underestimated for the morning and overestimated for the evening.

Assuming that the most efficient geocoronal absorption coefficient is the density of hydrogen at the exobase, the last result can be interpreted as an underestimate of the hydrogen density in the morning and as an overestimate in the evening. In other words, the hydrogen density estimated through the spherical model is an average dayside density, the morning density being higher than that average value and the evening lower. To evaluate this density gradient, we were led to compare an aspherical exospheric model with our measurements.

The study of orbits with a sun-orbit angle greater than $10^{\circ}$ was attempted. The results were very poor, because the average dayside density estimated by such a model is too large for measurements made at a greater angular distance from the solar direction, that is to say, from deeper in the geocorona. The increase in the hydrogen density is then artificial and is not induced by a decrease in solar activity. As all the other parameters are disturbed by this artificial change, all orbits having a sun-orbit angle greater than $10^{\circ}$ are excluded from this study.

Comparison with the Jacchia, Kockarts and Nicolet aspherical model. As was explained before, the measurements seem to contain little information about exospheric temperature. Therefore we decided to use an aspherical model in which the exospheric temperature would be taken as a fixed parameter. The model used is the one previously described, and the exospheric temperature chosen is that of Jacchia [1971].

It is possible in such a model to introduce 
Vidal-Madjar et al.: Solar Lyman $\alpha$ and Hydrogen Exobase

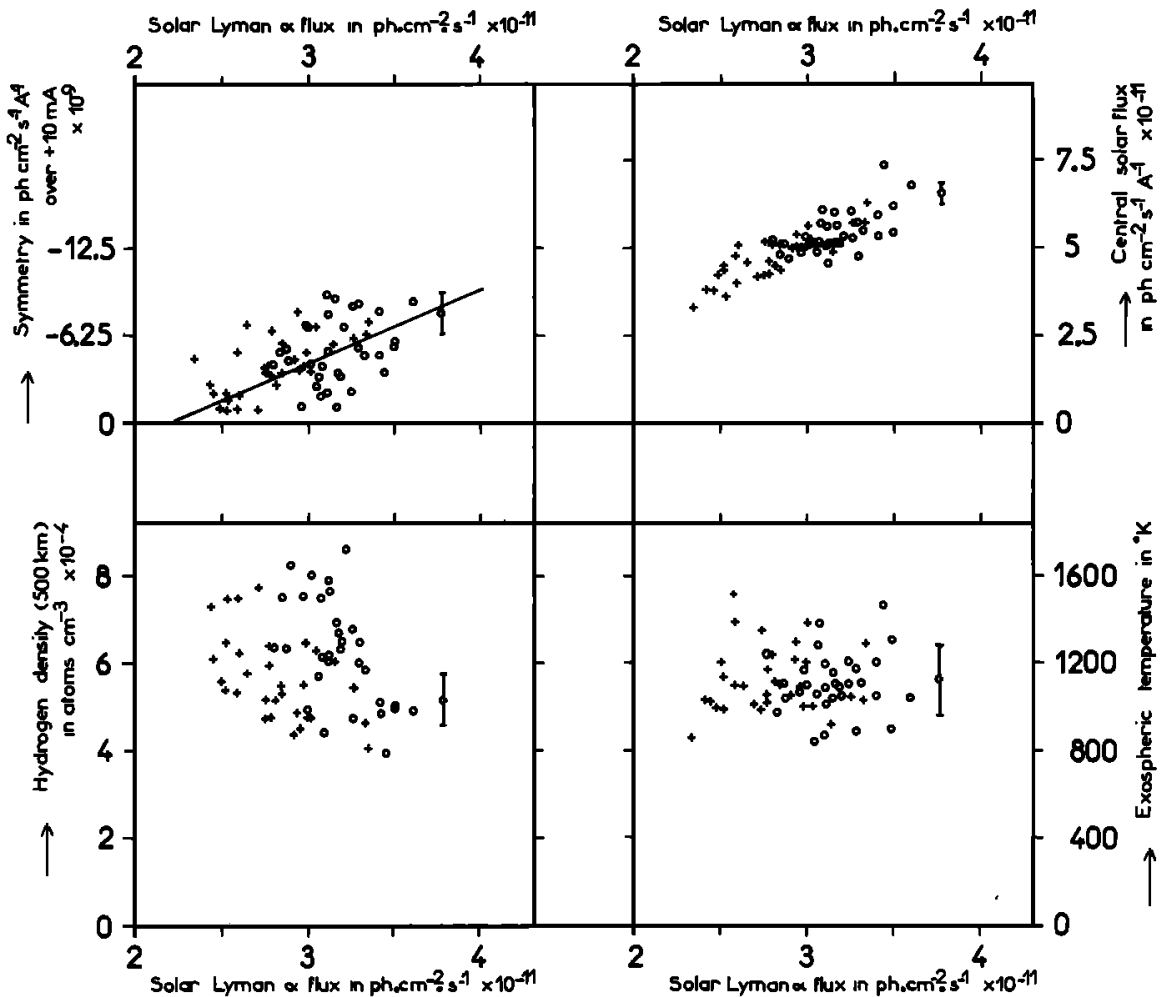

Fig. 11. Correlation of a four-parameter fit (flat solar center, spherical exosphere) with the total Lyman $\alpha$ solar flux as measured by the experiment: circles, 1969; crosses, 1970.

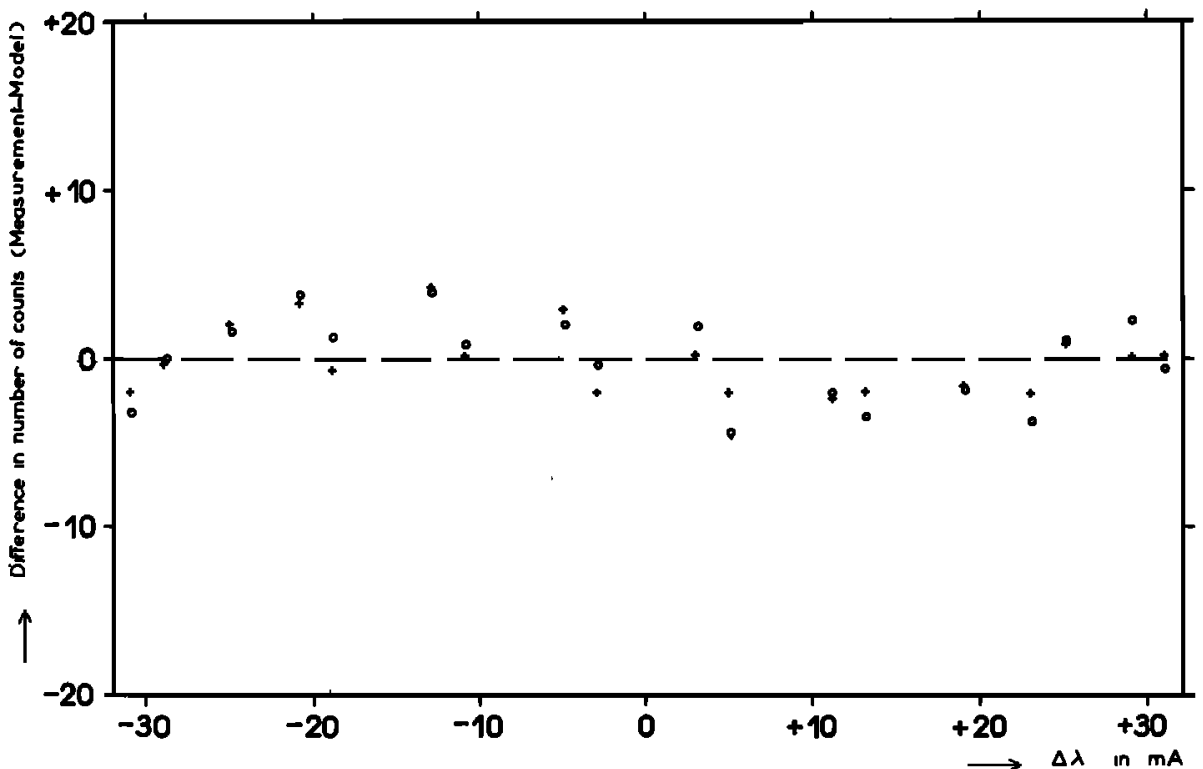

Fig. 12. Deviation from a perfect fit of a four-parameter model (flat solar center, spherical exosphere): circles, 1969; crosses, 1970. 
a hydrogen density distribution at the exobase. The Jacchia [1971] approximation of the Kockarts and Nicolet [1963] distribution is used, since it is more convenient than the original.

As can be seen in Figure 13, the systematic differences between the measurements and that model are very large, showing that certainly one of the fixed geocoronal values is wrong. It seems obvious that the temperature alone is not able to create such differences. Thus the Kockarts and Nicolet density distribution is found to be responsible. It is therefore abandoned in the following study as a good model of the diurnal variation of the atomic hydrogen density at the exobase.

Comparison with the Jacchia nonspherical model. The Jacchia [1971] exospheric temperature is used in the nonspherical model, as well as a density distribution depending on two parameters, $H_{\min }$ and $H_{\max }$. The density variation determined between day and night is roughly a factor of 2 , confirming several observations of the earth's exobase made by various authors using different techniques: observation of the Lyman a geocoronal emission [Meier, 1969b; Metzger and Clark, 1970], observation of the Balmer and geocoronal emission [Tinsley, 1970], and in situ measurements of hydrogen concentrations [Brinton and Mayr, 1971]. How- ever, such a model still presents strong systematic differences, the measurements showing that some of its constraints are probably not realistic (see Figure 13). Therefore, in the following study, a more flexible model is used.

Comparison with models having axial symmetry. The fifth model has a symmetry axis whose direction is defined by the couple $\left(\alpha_{\mathrm{Bym}}\right.$, $\left.\delta_{\text {sym }}\right)$. The temperature and density variations are sinusoidal. The minimum temperature coincides with the maximum density just at the point located at the exobase in the $\left(\alpha_{\mathrm{gym}}, \delta_{\mathrm{gym}}\right)$ direction. As is shown in (8) the temperature varies between $T_{\min }$ and $T_{\max }$, and the density between $H_{\max }$ and $H_{\mathrm{m} \mid \mathrm{m}}$.

In the axial symmetry model the $T_{\min }$ exospheric temperature is equal to the $T_{0}$ temperature as defined by Jacchia [1971]. A first study is made with such a model in order to fix the values of some of the parameters. This study is completed by observing how a change in the value of a parameter induces variations in the systematic differences between measurements and model.

The first study concerns the $\alpha_{\mathrm{sym}}$ parameter, the $\delta_{\text {aym }}$ value always being equal to the solar declination.

As is shown in Figure 14, assuming that the solar reversal is flat and horizontal (only one

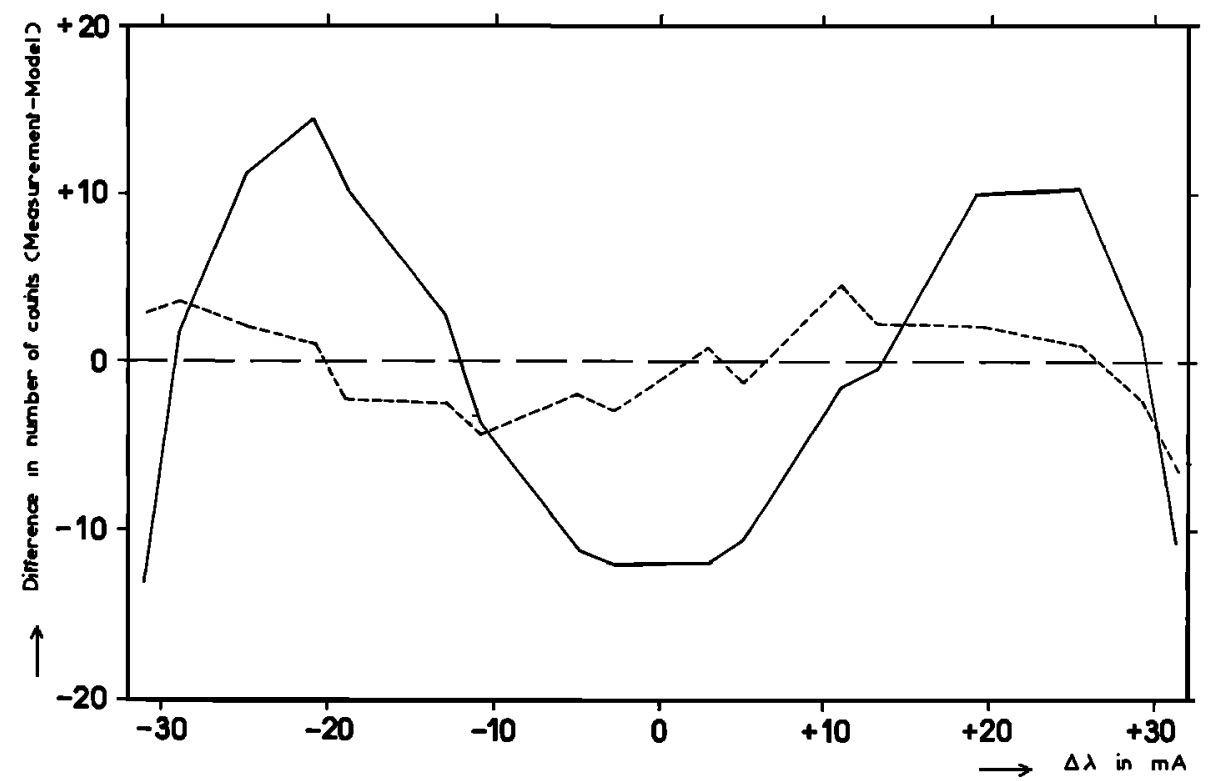

Fig. 13. Deviation from a perfect fit of two aspherical models (flat solar center) : solid line, Jacchia, Kockarts and Nicolet model ; dashed line, Jacchia nonspherical model. 


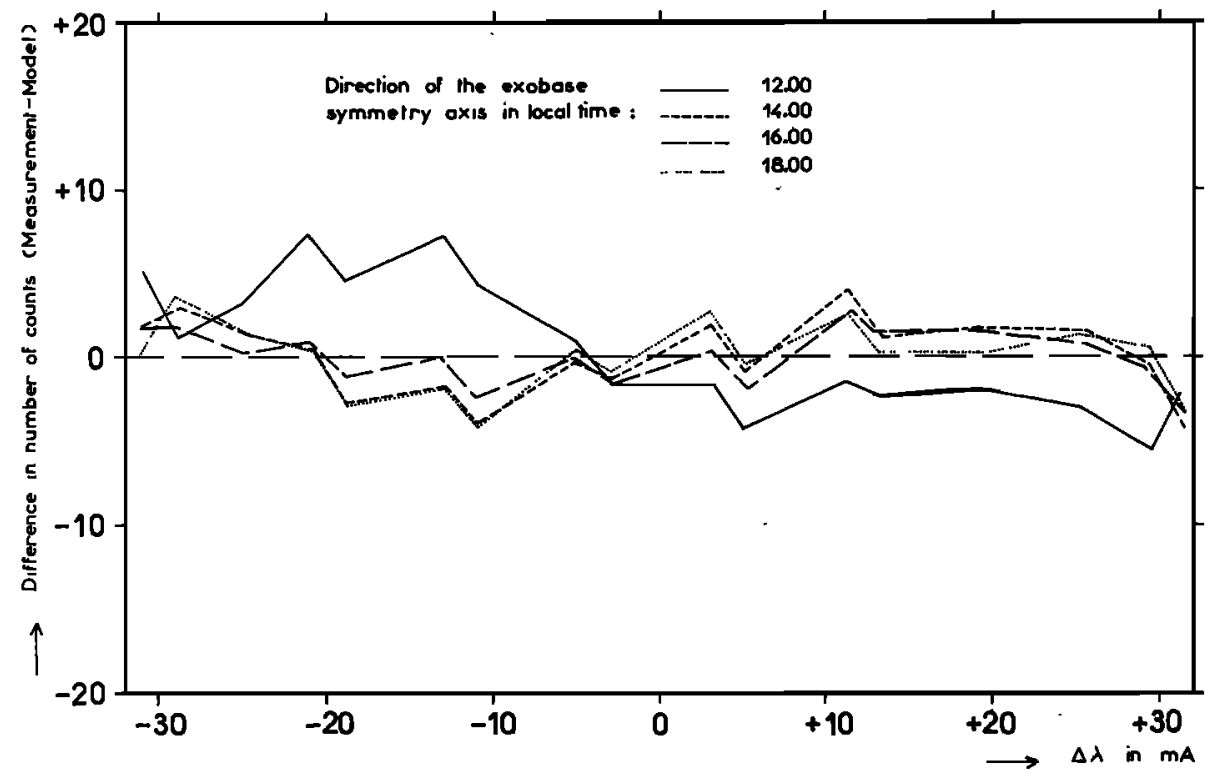

Fig. 14. Influence of the direction of the exaspheric model's symmetry axis on the quality of the fit (flat and horizontal solar center).

parameter $c$ ), for different values of the direction of the symmetry axis in local time (1200, $1400,1600,1800)$, the systematic differences are studied. The worst model is for $\boldsymbol{\alpha}_{\mathrm{sym}}=12.00$. The reason is, obviously, that in such a model no parameter can take into account the morning-evening dissymmetry already observed: the solar reversal is horizontal and flat, and the exobase is symmetric. The best fit is obtained for $\alpha_{s y m}=16.00$, which may indicate a possible later position of the maximum temperature. This result, although in agreement with the incoherent scattering observations [Bauer et al., 1970], does not give an unambiguous value of the local time of the maximum of temperature for two reasons: (1) The two other models with $\alpha_{\text {sym }}$ equal to 1400 and 1800 also seem to describe the observations. (2) As was explained above, this experiment is quite insensitive to the exospheric temperature, indicating a possible direction of the minimum density at around 16.00 without necessarily placing the direction of the maximum temperature at the same place. It could certainly be at 1400 , as in Jacchia's exospheric temperature model.

To show again how insensitive this experiment is to exospheric temperature, a study was made of the $T_{\max } / T_{\min }$ ratio effect in the best fit situation previously found $\left(\alpha_{s y m}=16.00\right.$ ). As is shown in Figure 15, systematic differences are present for three models having ratios of $1.1,1.3$, and 1.5. The best fit is found for the 1.3 ratio, as defined by Jacchia [1971]. Nevertheless, this is a rather poor confirmation of Jacchia's result.

A third study was made in order to evaluate the effect of the solar center line slope ( $b$ parameter). We were obliged to separate this parameter from the classical least squares study because it has a role equivalent to the density gradient (ratio between $H_{\min }$ and $H_{\text {max }}$ ). This slope was evaluated according to the first study made, and is linearly related, as the center solar flux, to the solar Lyman $\alpha$ total solar flux following the straight line drawn in Figure 11.

Under such conditions, we again tried models with various values of $\alpha_{a y m}: 1200,1400,1600$, and 1800 . The differences between measurements and models are presented in Figure 16. The 'worse fit is again for $\alpha_{\text {gym }}=1200$, but it appears to be considerably better than the fit obtained with the model using a flat and horizontal solar center, since this $b$ parameter takes care of part of the asymmetry. Again, the model with $a_{\mathrm{aym}}=16.00$ is the best, but the two other solutions are even closer than in the 


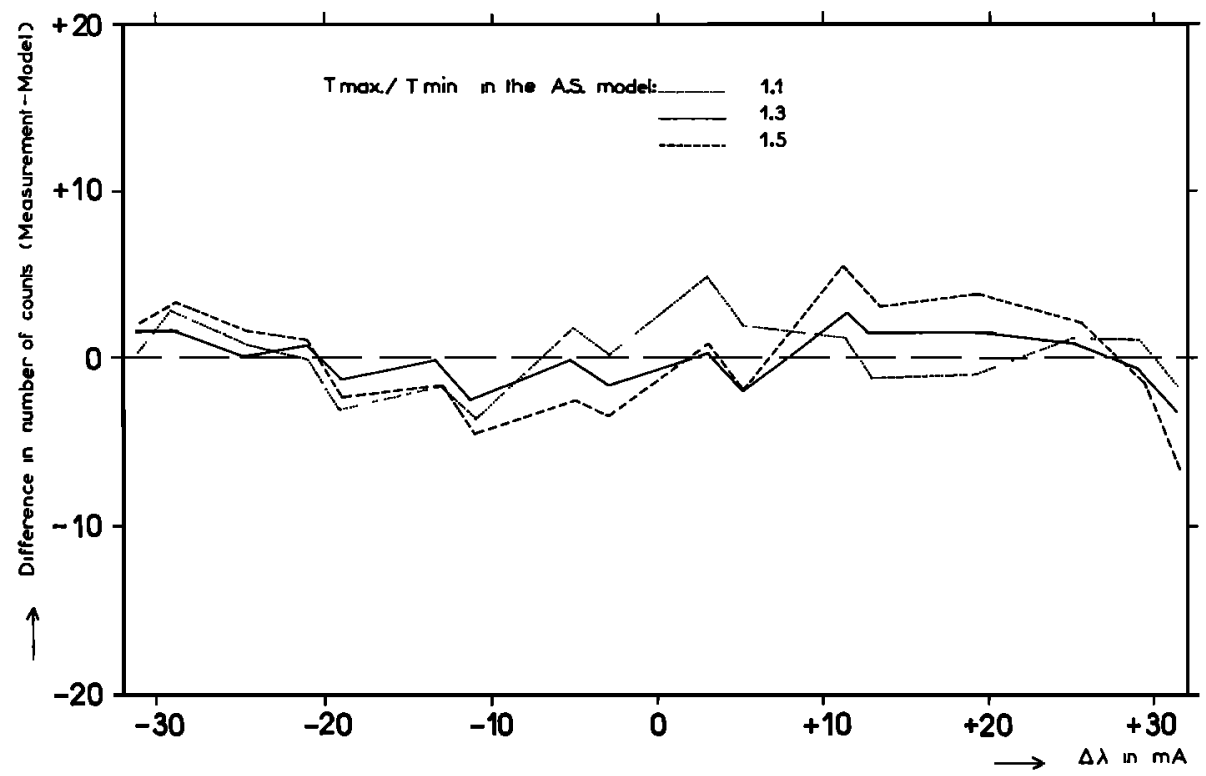

Fig. 15. Influence of the exospheric temperature ratio $T_{\max } / T_{\min }$ on the quality of the fit. (A. S. model : model having axial symmetry.)

fit previously obtained. This is why, henceforth, we will use in the axial symmetry model the fixed parameters as defined in the best solution found. symmetry model. Solar and geocoronal parameters fixed by the previous best solutions are as follows: $a=0$ (flat); $b$ is a linear function of the total solar flux as shown in Figure 11; $T_{\min }=T_{\mathrm{o}}(\mathrm{Jacchia}) ; T_{\max }=1.3 \times T_{\min }$;

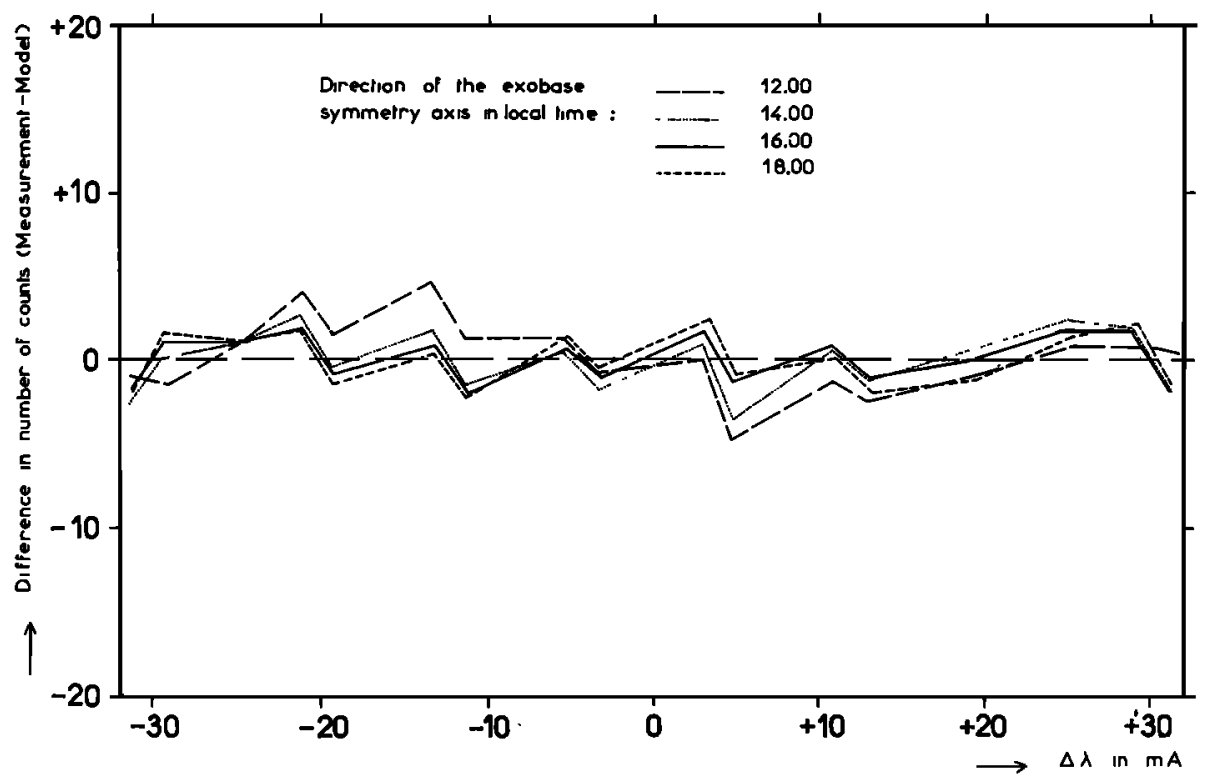

Fig. 16. Influence of the direction of the exospheric model's symmetry axis on the quality of the fit (flat and sloping solar center). 
$\alpha_{\mathrm{aym}}=16.00 ; \delta_{\mathrm{gym}}=\delta_{\mathrm{s}}$. Only three parameters are still free, and these will be found by the nonlinear least squares technique: the solar parameter $c$, which is the solar flux at the center of the Lyman $\alpha$ line; and the geocoronal parameters $H_{\min }$ and $H_{\max }$, which are the extreme values of the atomic hydrogen density at the exobase.

The superiority of this model over the spherical one was immediately observed: it is possible to take into account all the orbits of measurement with such a model. The reason is that, when an orbit of measurement is deep inside the geocorona, we will not observe any artificial increase in the values of $H_{\mathrm{m} \text { In }}$ and $H_{\mathrm{max}}$, since the model takes into account the position of the orbit and affects the measurements by means of the correct geometric coefficients.

Nevertheless, because one orbit having a sun-orbit angle greater than $25^{\circ}$ shows a rather small number of counts (see Figure 3 ) and because, at the limit, measurements made during one orbit whose plane is $90^{\circ}$ from the sun do not contain any information about the daynight density gradient, it was decided to analyze only those orbits with a sun-orbit angle $<25^{\circ}$. Studying other orbits will not give any new information but, on the contrary, will confuse the results by introducing more imprecision.

The quality of such a model can be immediately evaluated by examining the systematic differences between the measurements and the model (Figure 17). Averaged over 1 year, these differences are negligible. The systematic differences found over the year 1969 are exactly the same as those for 1970 (the reason only one curve was drawn in Figure 17). Even these very small differences are stable and therefore may contain some information.

The first important result concerns the solar flux at the center of the Lyman $\alpha$ line. As presented in Figure 18, the correlation coefficient of this flux with the total Lyman $\alpha$ solar flux is equal to 0.87 and confirms the result already found with the spherical model. The high quality of this correlation again confirms the quality of the data obtained through two completely independent channels. A typical error bar indicates that the linear relation drawn in Figure 18 is an excellent evaluation of the solar flux at the center of the Lyman $\alpha$ line. This linear relation shows how a $30 \%$ variation of the total Lyman $\alpha$ flux, as observed, corresponds to a $47 \%$ change at the center of the line. The decay

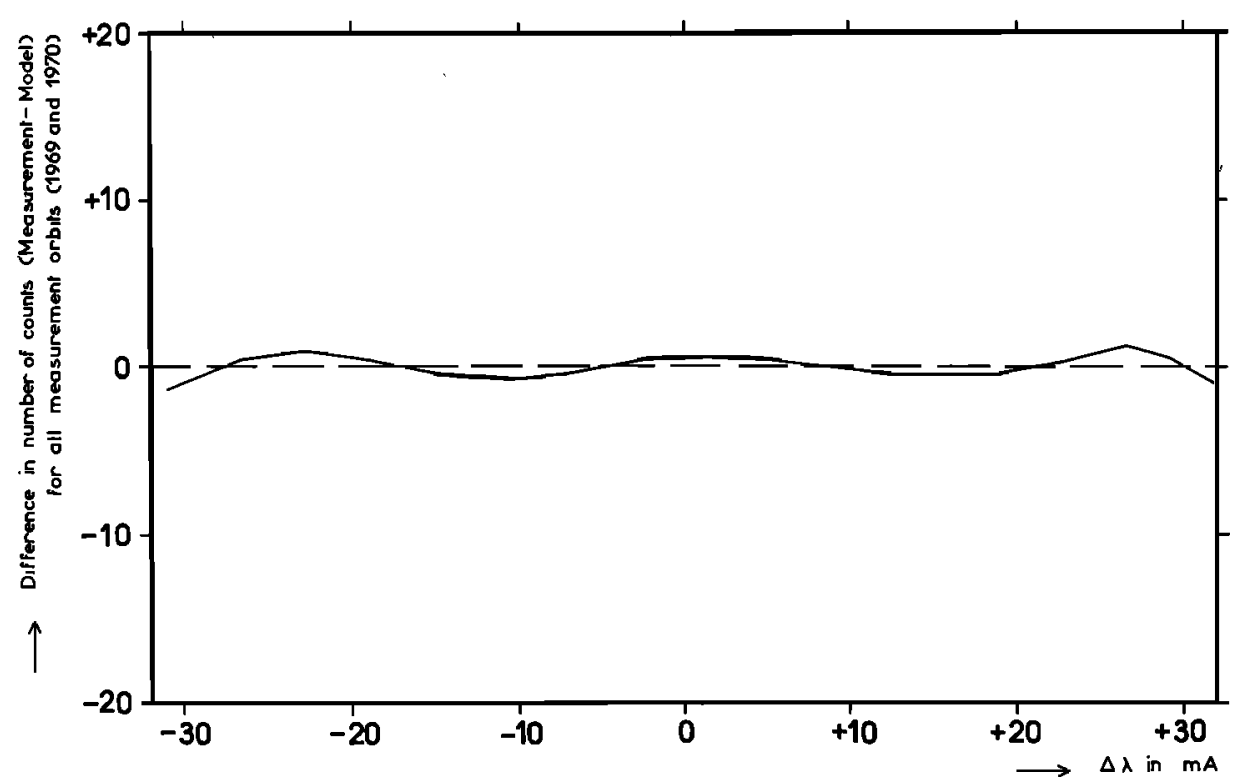

Fig. 17. Deviation from the perfect fit of the best aspherical exospheric model (flat and sloping solar center); all orbits are considered here. Direction of the geocoronal symmetry axis is $1600 ; T_{\operatorname{mex}}=1.3 T_{\min }$. 


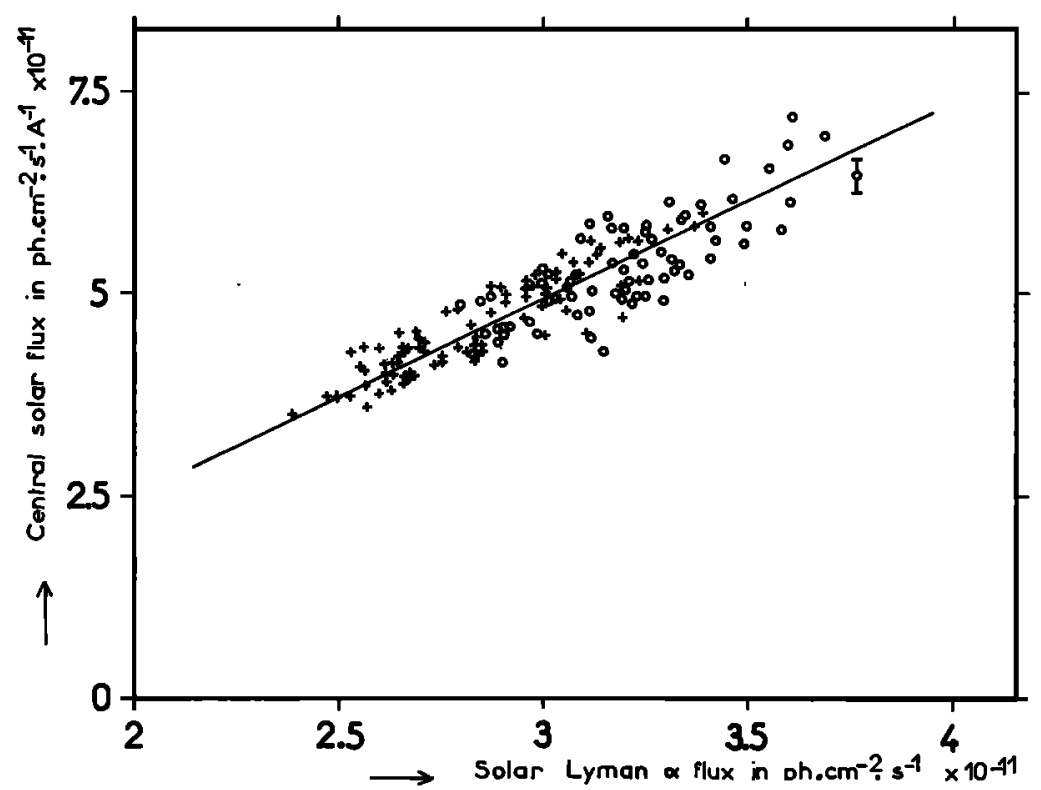

Fig. 18. Correlation of the flux at the center of the Lyman $\alpha$ line with the total line flux for the 1969 (circles) and 1970 (crosses). Only orbits with sun-orbit angle $<25^{\circ}$ were analyzed.

of the total and central solar fluxes, as measured from 1969 to 1970, seems to be caused, mainly, by a decrease in solar activity rather than by a weakening of the experiment's transmission. In effect, the latter case would result in an equal decrease in both fluxes rather than the maintenance of the straight line observed. Therefore we can say that the decrease in sensitivity of the experiment from one year to the other is rather small, roughly of the order of $10 \%$. In Table 2 are presented these results as a function of the 1969 and 1970 days (for orbits having a sun-orbit angle $<25^{\circ}$ ).

The other results obtained through this model concern the two exospheric parameters $H_{\mathrm{m} 1 \mathrm{n}}$ and $H_{\text {max. }}$. Because these two parameters are related to geocoronal conditions, their variations are represented as functions of the corresponding exospheric temperatures. In Figure 19, these results are given for the year 1969; the results for 1970 are very similar, and so are omitted for clarity. Results for both years are presented in Table 3, along with the density ratio $H_{\max } / H_{\min }$.

Confirming the result found with the Jacchia nonspherical model, this ratio is again roughly equal to 2. In Figure 19 the minimum and maximum densities of atomic hydrogen are absolute values, independent of the calibration of the experiment. Therefore these values are directly compared with the Kockarts and Nicolet [1963] distribution in order to show that this theoretical model underestimates $H_{\min }$ considerably and $H_{\max }$ slightly. Because the experiment is not very sensitive to temperature, one could say that the misfit comes from choosing the wrong exospheric temperature [Jacchia, 1971]. To improve the quality of the fit, $T_{\mathrm{m} 1 \mathrm{n}}$ must be decreased by about $20^{\circ} \mathrm{K}$ and $T_{\max }$ by about $200^{\circ} \mathrm{K}$, simultaneously completely changing the $T_{\max } / T_{\min }$ ratio. This second explanation has to be rejected, as it presupposes that the temperature of the atomic hydrogen is far from the exospheric temperature of the other constituents. Furthermore, the $T_{\max } / T_{\operatorname{mln}}$ ratio defined by Jacchia concurs with our data, as was already shown.

Throughout this study the geomagnetic heating of the atmosphere (as defined by Jacchia [1971]) has not been taken into account for two main reasons: first, because all our measurements are made in the equatorial regions (the orbit's inclination is $33^{\circ}$ ), and, second, because what we need in our study is not a precise 
Vidal-Madjar et al.: Solar Lyman $\alpha$ and Hydrogen Exobase

TABLE 2. Central Solar Lyman $\alpha$ Flux $\times 10^{11}$ $\mathrm{ph} \mathrm{\textrm {cm } ^ { - 2 }} \mathrm{sec}^{-1} \mathrm{~A}^{-1}$

\begin{tabular}{ll}
\hline Day & Flux \\
\hline
\end{tabular}

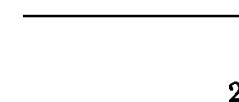

26.63

30.35

30.75

34.47

36.32

40.18

44.23

52.07

72.66

76.38

82.29

84.28

102.08

106.79

112.63

114.63

118.54

120.54

122.53

124.52

126.45

130.43

152.08

154.80

156.79

158.72

160.71

162.63

166.55

169.54

172.46

176.37

202.14

204.79

206.78

208.71

210.70

212.62

214.62

216.54

220.59

222.51

224.51

226.43

228.42

230.35

248.14

250.06

260.68

272.57

276 . 48

278.47

280.33

282.26
5.91

5.85

5.95

6.64

6.52

6.15

5.48

6.45

5.77

7.18

6.95

6.11

6.11

6.84

5.68

5.69

6.09

5.95

5.82

5.22

5.37

5.36

4.46

4.92

5.48

5.82

5.81

5.42

5.14

4.89

4.98

4.39

4.46

4.54

4.87

4.95

5.15

4.95

5. 38

5.20

5.15

5.27

4.93

4.61

4.49

4.23

4.95

4.22

4.71

5.31

4.75

5.02

4.41

4.86
TABLE 2. (continued)

\begin{tabular}{ll}
\hline Day & Flux \\
\hline 286.17 & 4.59 \\
288.16 & 4.59 \\
292.15 & 4.13 \\
294.14 & 5.01 \\
296.13 & 5.67 \\
301.04 & 5.60 \\
326.39 & 5.74 \\
328.32 & 5.79 \\
330.31 & 5.67 \\
332.23 & 5.07 \\
334.22 & 5.12 \\
338.14 & 5.15 \\
340.13 & 5.23 \\
342.12 & 4.93 \\
344.11 & 4.85 \\
348.09 & 4.89 \\
350.08 & 5.28 \\
352.00 & 5.29
\end{tabular}

5.09

5.65

5.78

5.80

6.02

5.63

5. 37

5.15

5.22

5.17

4.34

4.47

4.67

5.15

5.21

5.06

5.21

5.47

5.52

5.46

68.29

4.48

5.37

5.07

5.47

4.97

4.87

4.90

5.66

5.63

5.03

102.52

4.65

104.50

106.49

112.33

114.32

136.81

139.06

140.72
4.29

4.50

3.94

4.94

5.03

4.83 
TABLE 2. (continued)

\begin{tabular}{cc}
\hline Day & Flux \\
\hline 142.77 & 4.30 \\
144.70 & 4.17 \\
146.62 & 4.32 \\
150.53 & 4.78 \\
154.45 & 4.60 \\
158.36 & 4.32 \\
160.35 & 4.40 \\
184.02 & 5.07 \\
186.02 & 4.97 \\
188.00 & 4.81 \\
191.71 & 4.49 \\
193.64 & 4.12 \\
195.63 & 3.89 \\
197.62 & 3.87 \\
199.54 & 3.87 \\
201.53 & 4.11 \\
203.45 & 4.24 \\
205.44 & 4.26 \\
209.35 & 4.76 \\
211.34 & 4.75 \\
233.09 & 4.40 \\
235.01 & 4.49 \\
239.79 & 4.27 \\
241.77 & 3.96 \\
243.76 & 3.96 \\
243.96 & 3.94 \\
249.60 & 4.16 \\
251.52 & 4.10 \\
253.45 & 4.07 \\
255.43 & 3.98 \\
257.42 & 3.70 \\
259.35 & 3.69 \\
261.33 & 3.79 \\
265.31 & 4.08 \\
279.17 & 4.02 \\
287.92 & 4.10 \\
315.23 & 3.83 \\
317.29 & 4.24 \\
319.21 & 4.34 \\
321.20 & 4.27 \\
323.12 & 4.32 \\
325.11 & 4.29 \\
327.10 & 4.26 \\
329.09 & 4.16 \\
331.14 & 4.30 \\
333.06 & 4.30 \\
357.39 & 3.77 \\
359.31 & 3.66 \\
361.30 & 3.49 \\
365.21 & 3.59 \\
\hline &
\end{tabular}

exospheric temperature (to which our data are not sensitive) but a good atmospheric index following closely all exospheric modifications. As a matter of fact, we have found in the total Lyman $\alpha$ solar flux as measured by the experi- ment an index describing the variations of the solar Lyman $\alpha$ line center. What we needed here was a clear and simple index for the geocorona. Jacchia's minimum exospheric temperature $T_{0}$ answered this question, providing an excellent correlation with $H_{\max }$ and $H_{\min }$. An evaluation of the hydrogen density at the exobase is now quite simple for any given day, just because one can calculate the Jacchia $T_{0}$ temperature of that particular day and then deduce, through the use of Figure 19, the corresponding densities. Nevertheless, it is not impossible that the use of the Jacchia [1971] exospheric temperature corrected for the geomagnetic effect could reduce the scatter in Figure 19, showing then the quality of the Jacchia geomagnetic correction, along with its physical meaning.

\section{Discussion}

This discussion will refer mainly to the results obtained using the axial symmetry model. The main solar result obtained concerns the 47\% variation observed at the center of the Lyman $\alpha$ line. Meier [1969a] deduced a 25\% variation from observations of the geocoronal Lyman $\alpha$ emission. Nevertheless, these two values do not conflict, because Meier's value of $25 \%$ is certainly an underestimation of the real variation of the solar line's center. In effect, the geocoronal hydrogen density decreases with increasing solar flux, and consequently the geocoronal emission increases less than does the solar line's center. This is also why direct observations of the center of the solar Lyman $\alpha$ line show $100 \%$ variations, as was already explained by Blamont and Vidal-Madjar [1971].

On the other hand, this model yielded solutions that defined an exobase with a density ratio $\left(H_{\max } / H_{\min }\right)$ roughly equal to 2 . The Kockarts and Nicolet [1963] absolute hydrogen density model predicts, in agreement with our data, that the maximum hydrogen density must be found on the night side of the earth. Godart and Nicolet [1963] showed that the time constant of the hydrogen redistribution is short enough to induce a density factor of 5 between night and day. Compared to our data, this ratio seems much too high.

Other relative exobase models were constructed to define the atomic hydrogen distribution at $500 \mathrm{~km}$. First, an approximate timedependent solution was constructed by Hanson 


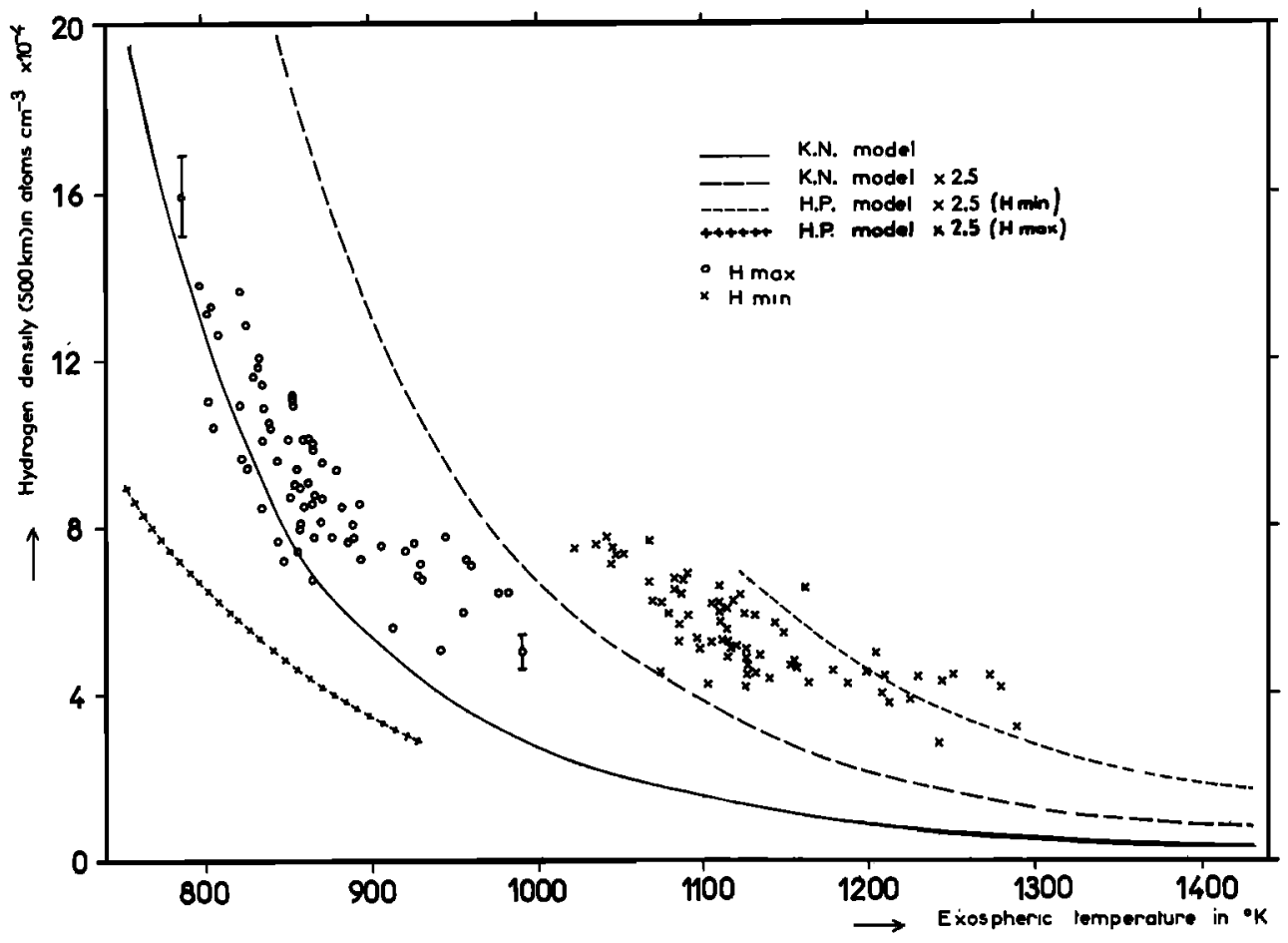

Fig. 19. Correlation of the minimum and maximum hydrogen densities of the exobase with the corresponding Jacchia exospheric temperatures ( $T_{\max }$ corresponds to $H_{\min }$ and $T_{\min }$ to $H_{\text {max }}$ ) for 1969 orbits with a sun-orbit angle $<25^{\circ}$. Values are compared with the corresponding densities as predicted by Kockarts and Nicolet [1963] and by Hanson and Patterson [1963] after normalization.

and Patterson [1963]. This solution, in good agreement with our data, presents roughly a ratio of 2 between $H_{\max }$ and $H_{\min }$ at $500 \mathrm{~km}$ altitude. As Figure 19 shows, this solution represents roughly the departure of $H_{\min }$ and $H_{\max }$ from the average steady state solution if this one is normalized by a 2.5 factor (as will be explained in a later section). In effect, $H_{\mathrm{m} \ln }$ is above the average steady state solution, and $H_{\max }$ is below, inducing a smaller $H_{\max } / H_{\min }$ ratio. Nevertheless, more recently, Wallace and Strobel [1972] calculated an exact solution of the time dependent equations in the thermosphere, neglecting also the lateral transport in the exosphere. They found a little larger density ratio between $H_{\min }$ and $H_{\max }$ than Hanson and Patterson did. This result is still in agreement with our data, as well as the location of the minimum of density at $500 \mathrm{~km}$, which they found around 1600 .

Second, Patterson [1966] and then McAfee [1967] took into account the lateral transport of hydrogen atoms in the earth's exosphere from one point of the exobase to another. McAfee has defined, for several temperature models, the bydrogen density distribution inducing in all points of the exobase the zero net ballistic flux conditions: ballistic flux going out is equal to ballistic flux coming in. Such a relative hydrogen distribution gives, again, roughly a factor of 2 between day and night. More recently, Quessette [1972] improved the technique defined by $M c$ Afee [1967] and studied more precisely the variation of the $H_{\max } / H_{\min }$ ratio as a function of the exospheric temperature model. He found a quasi-linear relation between the $H_{\mathrm{m} 1 \mathrm{n}} / H_{\max }$ ratio of a density distribution following the zero net ballistic flux condition and the representative value of the exospheric temperature model:

$$
\frac{T_{\max }-T_{\min }}{\left[\left(T_{\max }+T_{\min }\right) / 2\right]^{2}}=\Delta T / T^{2}
$$

Comparing our data to Quessette's relation, 
TABLE 3. Critical Level (500 km) Hydrogen Densities $\times 10^{4}$ atoms $\mathrm{cm}^{-3}$

\begin{tabular}{|c|c|c|c|c|c|}
\hline Day & $T_{\min }$ & $T_{\max }$ & $H_{\min }$ & $H_{\max }$ & $H_{\max } / H_{\min }$ \\
\hline \multicolumn{6}{|c|}{1969} \\
\hline 26.63 & 859. & 1117. & 5.02 & 8.46 & 1.68 \\
\hline 30.35 & 853. & 1109 & 5.64 & 11.02 & 1.95 \\
\hline 30.75 & 853. & 1109 & 5.86 & 10.92 & 1.86 \\
\hline 34.47 & 865. & 1125 & 4.09 & 6.76 & 1.65 \\
\hline 36.32 & 865. & 1125 & 4.42 & 8.54 & 1.93 \\
\hline 40.18 & 866. & 1125. & 4.61 & 8.70 & 1.89 \\
\hline 44.23 & 858. & 1115. & 5.03 & 8.01 & 1.59 \\
\hline 52.07 & 905 & 1177 & 4.47 & 7.55 & 1.69 \\
\hline 72.66 & 883. & 1147 & 5.37 & 8.46 & 1.58 \\
\hline 76.38 & 931. & 1210 & 3.72 & 6.73 & 1.81 \\
\hline 82.29 & 990. & 1287. & 3.12 & 4.96 & 1.59 \\
\hline 84.28 & 955. & 1241 & 2.75 & 5.91 & 2.15 \\
\hline 102.08 & 887. & 1154 & 4.68 & 7.65 & 1.63 \\
\hline 106.79 & 928. & 1206 & 3.90 & 6.80 & 1.75 \\
\hline 112.63 & 894. & 1162 & 4.18 & 7.20 & 1.72 \\
\hline 114.63 & 876. & 1139 & 4.29 & 7.72 & 1.80 \\
\hline 118.54 & 865. & 1125. & 4.68 & 7.75 & 1.66 \\
\hline 120.54 & 857. & 1114 & 5.16 & 7.93 & 1.54 \\
\hline 122.53 & 854 & 1110 & 6.47 & 8.95 & 1.38 \\
\hline 124.52 & 863. & 1121 & 6.20 & 10.11 & 1.63 \\
\hline 126.45 & 892 . & 1159 & 6.46 & 8.52 & 1.32 \\
\hline 130.43 & 865. & 1124 & 4.96 & 9.83 & 1.98 \\
\hline 152.08 & 821 & 1067. & 6.64 & 10.89 & 1.64 \\
\hline 154.80 & 832 . & 1081 & 6.71 & 11.84 & 1.76 \\
\hline 156.79 & 879 & 1143 & 5.61 & 9.37 & 1.67 \\
\hline 158.72 & 925. & 1203 & 4.87 & 7.60 & 1.56 \\
\hline 160.71 & 977 & 1270. & 4.35 & 6.38 & 1.47 \\
\hline 162.63 & 982 & 1277 & 4.06 & 6.41 & 1.58 \\
\hline 166.55 & 956 & 1242 . & 4.21 & 7.15 & 1.70 \\
\hline 169.54 & 871. & 1132 & 4.82 & 8.66 & 1.80 \\
\hline 172.46 & 856. & 1113 & 5.91 & 8.93 & 1.51 \\
\hline 176.37 & 839. & 1090 & 6.80 & 10.38 & 1.53 \\
\hline 202.14 & 808 & 1050 & 7.28 & $12: 59$ & 1.73 \\
\hline 204.79 & 803. & 1045 & 7.48 & 13.24 & 1.77 \\
\hline 206.78 & 801 & 1041 & 7.73 & 13.06 & 1.69 \\
\hline 208.71 & 802. & 1043 & 7.05 & 11.03 & 1.56 \\
\hline 210.70 & 821 & 1067 & 7.57 & 13.58 & 1.79 \\
\hline 212.62 & 835. & 1085 & 6.25 & 10.09 & 1.61 \\
\hline 214.62 & 870 . & 1131 & 5.76 & 9.52 & 1.65 \\
\hline 216.54 & 887. & 1153 & 4.60 & 7.67 & 1.67 \\
\hline 220.59 & 856. & 1112 & 5.17 & 7.45 & 1.44 \\
\hline 222.51 & 834 & 1085 & 5.60 & 8.45 & 1.51 \\
\hline 224.51 & 822. & 1069 & 6.12 & 9.62 & 1.57 \\
\hline 226.43 & 805. & 1047 & 7.29 & 10.39 & 1.43 \\
\hline 228.42 & 796. & 1034 & 7.47 & 13.75 & 1.84 \\
\hline 230.35 & 786. & 1022 & 7.41 & 15.90 & 2.15 \\
\hline 248.14 & 844. & 1098 & 5.08 & 7.64 & 1.50 \\
\hline 250.06 & 825. & 1073 & 4.48 & 9.40 & 2.10 \\
\hline 260.68 & 829 & 1077 & 5.87 & 11.58 & 1.97 \\
\hline 272.57 & 847 . & 1101 & 4.18 & 7.20 & 1.72 \\
\hline 276.48 & 850. & 1105 & 6.08 & 8.70 & 1.43 \\
\hline 278.47 & 836. & 1086 & 6.63 & 10.82 & 1.63 \\
\hline 280.33 & 850. & 1105 & 5.15 & 10.10 & 1.96 \\
\hline 282.26 & 859. & 1117 & 6.12 & 10.09 & 1.65 \\
\hline 286.17 & 834. & 1084 & 5.18 & 11.43 & 2.21 \\
\hline
\end{tabular}


TABLE 3. (continued)

\begin{tabular}{|c|c|c|c|c|c|}
\hline Day & $T_{\min }$ & $T_{\max }$ & $H_{\min }$ & $H_{\max }$ & $H_{\max } / H_{\min }$ \\
\hline $\begin{array}{l}288.16 \\
292.15 \\
294.14 \\
296.13 \\
301.04\end{array}$ & $\begin{array}{l}826 . \\
843 . \\
888 \\
920 \\
941\end{array}$ & $\begin{array}{l}1073 . \\
1095 . \\
1154 . \\
1197 . \\
1224 .\end{array}$ & $\begin{array}{l}6.15 \\
5.20 \\
4.55 \\
4.41 \\
3.76\end{array}$ & $\begin{array}{r}12.82 \\
9.55 \\
8.05 \\
7.42 \\
5.04\end{array}$ & $\begin{array}{l}2.09 \\
1.84 \\
1.77 \\
1.68 \\
1.34\end{array}$ \\
\hline $\begin{array}{l}326.39 \\
328.32 \\
330.31 \\
332.23 \\
334.22 \\
338.14 \\
340.13 \\
342.12 \\
344.11 \\
348.09 \\
350.08 \\
352.00\end{array}$ & $\begin{array}{l}944 . \\
960 . \\
929 . \\
913 . \\
870 . \\
856 . \\
857 . \\
839 \\
833 . \\
853 . \\
862 . \\
864 .\end{array}$ & $\begin{array}{l}1228 \\
1249 \\
1208 \\
1186 \\
1131 \\
1112 \\
1114 \\
1090 \\
1083 \\
1109 \\
1121 \\
1124\end{array}$ & $\begin{array}{l}4.32 \\
4.39 \\
4.31 \\
4.17 \\
4.45 \\
5.45 \\
4.83 \\
5.80 \\
6.37 \\
6.05 \\
6.23 \\
5.82\end{array}$ & $\begin{array}{r}7.71 \\
7.04 \\
7.12 \\
5.59 \\
8.13 \\
9.34 \\
8.85 \\
10.33 \\
12.00 \\
10.81 \\
9.00 \\
10.06\end{array}$ & $\begin{array}{l}1.79 \\
1.60 \\
1.65 \\
1.34 \\
1.83 \\
1.71 \\
1.83 \\
1.78 \\
1.88 \\
1.79 \\
1.44 \\
1.73\end{array}$ \\
\hline \multicolumn{6}{|c|}{1970} \\
\hline $\begin{array}{l}10.37 \\
12.36 \\
14.28 \\
16.27 \\
18.26 \\
20.18 \\
22.17 \\
26.16 \\
28.15 \\
30.14\end{array}$ & $\begin{array}{l}845 . \\
903 . \\
922 . \\
934 . \\
938 . \\
933 . \\
938 . \\
904 . \\
924 . \\
924 .\end{array}$ & $\begin{array}{l}1099 \\
1173 \\
1199 \\
1214 \\
1219 \\
1213 \\
1219 \\
1176 \\
1201 \\
1202\end{array}$ & $\begin{array}{l}6.03 \\
5.03 \\
4.49 \\
3.78 \\
3.77 \\
3.62 \\
3.81 \\
4.77 \\
5.06 \\
5.19\end{array}$ & $\begin{array}{r}\mathbf{1 0 . 4 9} \\
\mathbf{8 . 9 3} \\
\mathbf{7 . 5 8} \\
\mathbf{6 . 0 3} \\
\mathbf{6 . 2 4} \\
\mathbf{7 . 3 7} \\
\mathbf{5 . 9 8} \\
\mathbf{8 . 5 2} \\
\mathbf{8 . 3 7} \\
\mathbf{8 . 6 1}\end{array}$ & $\begin{array}{l}1.74 \\
1.78 \\
1.69 \\
1.59 \\
1.65 \\
2.04 \\
1.57 \\
1.79 \\
1.65 \\
1.66\end{array}$ \\
\hline $\begin{array}{l}35.04 \\
37.03 \\
38.76 \\
56.48 \\
58.40 \\
62.38 \\
64.37 \\
66.63 \\
66.76 \\
68.29\end{array}$ & $\begin{array}{l}870 . \\
865 . \\
879 . \\
955 . \\
945 . \\
941 . \\
931 . \\
934 . \\
934 . \\
940 .\end{array}$ & $\begin{array}{l}1131 \\
1124 \\
1142 \\
1242 \\
1229 \\
1223 \\
1210 \\
1215 \\
1215 \\
1222\end{array}$ & $\begin{array}{l}4.78 \\
6.07 \\
4.86 \\
\mathbf{3 . 5 5} \\
\mathbf{3 . 7 5} \\
\mathbf{4 . 5 1} \\
\mathbf{3 . 9 7} \\
\mathbf{3 . 7 4} \\
\mathbf{3 . 7 8} \\
2.96\end{array}$ & $\begin{array}{r}12.26 \\
10.61 \\
11.60 \\
5.76 \\
7.38 \\
7.15 \\
8.32 \\
7.39 \\
8.14 \\
4.50\end{array}$ & $\begin{array}{l}2.56 \\
1.75 \\
2.38 \\
1.62 \\
1.97 \\
1.58 \\
2.10 \\
1.98 \\
2.15 \\
1.52\end{array}$ \\
\hline $\begin{array}{r}80.16 \\
82.09 \\
84.07 \\
86.06 \\
87.99 \\
90.71 \\
94.69 \\
100.60 \\
100.66 \\
102.52\end{array}$ & $\begin{array}{l}894 . \\
907 . \\
930 . \\
923 . \\
913 . \\
896 . \\
913 . \\
984 . \\
984 . \\
968 .\end{array}$ & $\begin{array}{l}1162 . \\
1179 \\
1209 \\
1200 . \\
1187 \\
1165 \\
1187 \\
1280 \\
1280 \\
1258\end{array}$ & $\begin{array}{l}4.67 \\
4.63 \\
4.53 \\
4.07 \\
3.52 \\
3.16 \\
3.71 \\
3.29 \\
3.37 \\
3.78\end{array}$ & $\begin{array}{l}7.59 \\
7.78 \\
7.00 \\
7.88 \\
6.05 \\
6.80 \\
7.22 \\
6.05 \\
5.88 \\
6.27\end{array}$ & $\begin{array}{l}1.63 \\
1.68 \\
1.55 \\
1.94 \\
1.72 \\
2.15 \\
1.95 \\
1.84 \\
1.75 \\
1.66\end{array}$ \\
\hline $\begin{array}{l}104.50 \\
106.49 \\
112.33 \\
114.32 \\
136.81 \\
139.06 \\
140.72 \\
142.77 \\
144.70\end{array}$ & $\begin{array}{l}940 . \\
911 . \\
860 . \\
863 . \\
960 . \\
942 . \\
945 . \\
910 . \\
895 .\end{array}$ & $\begin{array}{l}1222 . \\
1184 . \\
1118 . \\
1122 . \\
1248 . \\
1225 . \\
1228 . \\
1183 . \\
1163 .\end{array}$ & $\begin{array}{l}4.44 \\
4.16 \\
4.80 \\
4.88 \\
4.58 \\
4.64 \\
4.20 \\
4.51 \\
4.12\end{array}$ & $\begin{array}{r}7.16 \\
6.90 \\
6.26 \\
10.07 \\
7.92 \\
7.53 \\
7.08 \\
8.59 \\
8.16\end{array}$ & $\begin{array}{l}1.61 \\
1.66 \\
1.30 \\
2.06 \\
1.73 \\
1.62 \\
1.69 \\
1.90 \\
1.98\end{array}$ \\
\hline
\end{tabular}


TABLE 3. (continued)

\begin{tabular}{|c|c|c|c|c|c|}
\hline Day & $T_{\min }$ & $T_{\max }$ & $H_{\min }$ & $\boldsymbol{H}_{\max }$ & $H_{\max } / H_{\min }$ \\
\hline $\begin{array}{l}146.62 \\
150.53 \\
154.45 \\
158.36 \\
160.35 \\
184.02 \\
186.02 \\
188.00 \\
191.71 \\
193.64\end{array}$ & $\begin{array}{l}891 . \\
889 . \\
868 . \\
852 . \\
857 . \\
908 . \\
915 . \\
911 . \\
857 . \\
842 .\end{array}$ & $\begin{array}{l}1159 . \\
1156 . \\
1128 . \\
1108 . \\
1114 . \\
1181 \\
1189 \\
1184 . \\
1114 . \\
1095\end{array}$ & $\begin{array}{l}4.90 \\
4.88 \\
5.11 \\
6.12 \\
5.70 \\
4.66 \\
4.61 \\
4.62 \\
4.81 \\
5.77\end{array}$ & $\begin{array}{r}8.94 \\
8.02 \\
9.06 \\
11.77 \\
11.04 \\
7.85 \\
7.23 \\
8.03 \\
10.19 \\
10.91\end{array}$ & $\begin{array}{l}1.83 \\
1.65 \\
1.77 \\
1.92 \\
1.94 \\
1.69 \\
1.57 \\
1.74 \\
2.12 \\
1.89\end{array}$ \\
\hline $\begin{array}{l}195.63 \\
197.62 \\
199.54 \\
201.53 \\
203.45 \\
205.44 \\
209.35 \\
211.34 \\
233.09 \\
235.01\end{array}$ & $\begin{array}{l}834 . \\
823 . \\
828 . \\
841 . \\
860 . \\
870 . \\
901 . \\
868 . \\
846 . \\
838 .\end{array}$ & $\begin{array}{l}1084 . \\
1070 . \\
1076 . \\
1093 . \\
1118 . \\
1131 . \\
1171 \\
1129 \\
1100 . \\
1090 .\end{array}$ & $\begin{array}{l}6.13 \\
6.20 \\
7.04 \\
7.11 \\
5.37 \\
5.09 \\
5.20 \\
4.85 \\
6.86 \\
6.52\end{array}$ & $\begin{array}{r}10.39 \\
12.00 \\
11.22 \\
9.51 \\
9.71 \\
7.99 \\
9.26 \\
10.03 \\
11.69 \\
12.33\end{array}$ & $\begin{array}{l}1.70 \\
1.93 \\
1.59 \\
1.34 \\
1.81 \\
1.57 \\
1.78 \\
2.07 \\
1.70 \\
1.89\end{array}$ \\
\hline $\begin{array}{l}239.79 \\
241.77 \\
243.76 \\
243.96 \\
249.60 \\
251.52 \\
253.45 \\
255.43 \\
257.42 \\
259.35\end{array}$ & $\begin{array}{l}830 . \\
839 . \\
849 . \\
849 . \\
863 . \\
865 . \\
855 . \\
826 . \\
809 . \\
809 .\end{array}$ & $\begin{array}{l}1080 . \\
1090 . \\
1104 . \\
1104 . \\
1122 . \\
1124 . \\
1111 . \\
1074 . \\
1052 . \\
1052 .\end{array}$ & $\begin{array}{l}5.25 \\
5.82 \\
5.08 \\
4.88 \\
5.31 \\
5.29 \\
5.25 \\
5.89 \\
5.36 \\
6.14\end{array}$ & $\begin{array}{r}12.19 \\
10.67 \\
10.90 \\
10.93 \\
10.02 \\
9.12 \\
9.47 \\
11.32 \\
9.50 \\
12.25\end{array}$ & $\begin{array}{l}2.32 \\
1.83 \\
2.14 \\
2.24 \\
1.89 \\
1.72 \\
1.80 \\
1.92 \\
1.77 \\
2.00\end{array}$ \\
\hline $\begin{array}{l}261.33 \\
265.31 \\
279.17 \\
287.92 \\
315.23 \\
317.29 \\
319.21 \\
321.20 \\
323.12 \\
325.11\end{array}$ & $\begin{array}{l}811 . \\
858 . \\
846 . \\
849 . \\
884 . \\
908 . \\
936 . \\
946 . \\
939 . \\
918 .\end{array}$ & $\begin{array}{l}1055 . \\
1115 . \\
1099 . \\
1104 . \\
1149 . \\
1180 . \\
1217 . \\
1229 . \\
1221 . \\
1194 .\end{array}$ & $\begin{array}{l}\mathbf{6 . 1 1} \\
\mathbf{4 . 6 2} \\
\mathbf{5 . 4 0} \\
\mathbf{4 . 0 6} \\
\mathbf{3 . 6 6} \\
\mathbf{4 . 3 9} \\
\mathbf{4 . 0 1} \\
\mathbf{3 . 6 1} \\
\mathbf{3 . 4 6} \\
\mathbf{4 . 3 6}\end{array}$ & $\begin{array}{r}13.91 \\
9.57 \\
8.75 \\
10.52 \\
8.64 \\
8.74 \\
6.99 \\
7.30 \\
6.37 \\
7.75\end{array}$ & $\begin{array}{l}2.28 \\
2.07 \\
1.62 \\
2.59 \\
2.36 \\
1.99 \\
1.74 \\
2.02 \\
1.84 \\
1.78\end{array}$ \\
\hline $\begin{array}{l}327.10 \\
329.09 \\
331.14 \\
333.06 \\
357.39 \\
359.31 \\
361.30 \\
365.21\end{array}$ & $\begin{array}{l}892 . \\
866 . \\
861 . \\
888 . \\
877 . \\
860 . \\
855 . \\
858 .\end{array}$ & $\begin{array}{l}1160 . \\
1125 . \\
1119 . \\
1154 . \\
1141 . \\
1118 . \\
1112 . \\
1116 .\end{array}$ & $\begin{array}{l}4.71 \\
5.10 \\
5.72 \\
5.02 \\
5.73 \\
5.54 \\
5.86 \\
6.33\end{array}$ & $\begin{array}{r}7.12 \\
8.93 \\
10.61 \\
10.38 \\
9.26 \\
10.19 \\
11.99 \\
9.88\end{array}$ & $\begin{array}{l}1.51 \\
1.75 \\
1.85 \\
2.07 \\
1.62 \\
1.84 \\
2.04 \\
1.56\end{array}$ \\
\hline
\end{tabular}

we plot (Figure 20) all the $H_{\max } / H_{\min }$ ratios obtained during the year 1969 as functions of $\Delta T / T^{2}$. Again the 1970 results are so similar that they are eliminated for the sake of clarity. The line representing the Quessette relation is drawn through the data points; it is surprising to see how well this straight line fits the results.
For comparison, the density ratio $\left(H_{\max } / H_{\min }\right.$ at $500 \mathrm{~km}$ ) calculated for the time-dependent solution in the thermosphere by Wallace and Strobel [1972], who neglected the lateral transport of hydrogen in the exosphere, is also represented in Figure 20.

From these two studies, one neglecting the 
diffusion from the thermosphere and the other neglecting the lateral transport in the exosphere, it appears that the density distribution at the exobase is controlled by the zero net ballistic flux condition, mainly because the ballistic flux is several orders of magnitude greater than the hydrogen source, as was explained by Hanson and Patterson [1963] and by McAfee [1967]: the ballistic flux is of the order of $10^{10} \mathrm{~cm}^{-2} \mathrm{sec}^{-1}$; the hydrogen source flux coming from the thermosphere, equivalent to the escape flux, is of the order of $10^{7} \mathrm{~cm}^{-2} \mathrm{sec}^{-1}$. This is why, as was already suggested by Quessette, in the thermosphere the time-dependent equations can be solved step by step, starting from the top, where the boundary distribution is fixed by the zero net ballistic flux condition.

Nevertheless, one should be very careful about this subject, mainly because some other source or sink of atomic hydrogen may exist in the exobase region. In particular, as Hanson and Patterson [1963] proposed, there is a possible source of hydrogen atoms during the night provided through charge exchange of the downward protons. Tinsley [1973] suggested that the effects of interactions with the ionosphere increase the diurnal ratio of about 1.4 calculated by Wallace and Strobel [1972] to near the observed value of 1.7 . Our data may then be consistent with Wallace and Strobel's model corrected for the previous effect.

Nevertheless, because the proton fluxes are certainly smaller than $10^{\circ} \mathrm{cm}^{-2} \mathrm{sec}^{-1}$ and probably of the order of $10^{8} \mathrm{~cm}^{-2} \mathrm{sec}^{-1}$, it is possible to evaluate the effect of such sources and sinks of atomic hydrogen by adding, in a McAfee [1967] or Quessette [1972] type calculation, a constant upward and downward flux of the previous magnitude to the corresponding upward and downward ballistic flux at particular locations of the exobase. To reach the zero net flux condition all over the exobase, these added fluxes induce density changes at the location where they are applied. This local density variation is of the order of the ratio between the ballistic flux and the proton flux, i.e., is of the order of $10 \%$ for a $10^{\circ} \mathrm{cm}^{-2} \mathrm{sec}^{-1}$ proton flux and of the order of $1 \%$ for a $10^{8} \mathrm{~cm}^{-2} \mathrm{sec}^{-1}$ proton flux.

Ho and Moorcroft [1971] explained that a $10^{\circ} \mathrm{cm}^{-2} \mathrm{sec}^{-1}$ flux is related to high measurements of the atomic hydrogen density, larger than our own measurements by about a factor

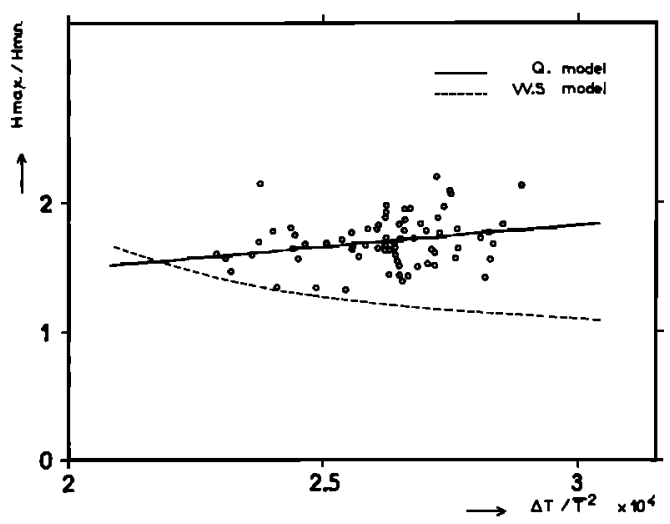

Fig. 20. Comparison of the density ratio $H_{\max } / H_{\min }$ for 1969 orbits with a sun-orbit angle $<25^{\circ}$ with the theoretical relation found by Quessette [1972] for the exobase distribution controlled by the zero net ballistic flux condition and with the ratio calculated by Wallace and Strobel [1972] in a time-dependent thermospheric model.

of 10 . If we assume that our density measurement is correct (independent of the experiment's calibration), we then can state that a more probable value of the proton flux in that region is of the order of $10^{9} \mathrm{~cm}^{-2} \mathrm{sec}^{-1}$. Under such conditions the correction to the McAfee or Quessette model is certainly very small, showing then that the most important effect that controls the density distribution at the exobase level is the zero net ballistic flux, mainly because the ballistic fluxes are 2 orders of magnitude larger than any other fluxes. Nevertheless, one must keep in mind that such a process is probably more important in middle- and high-latitude regions and may seriously affect the hydrogen density distribution.

In conclusion, it is possible to say that the Wallace and Strobel model, corrected for the proton flux effect, induces a 1.7 hydrogen density ratio. Under such a condition the exobase is then also in the zero net ballistic flux situation. Consequently, the ratio of 1.7 is of course not modifiable by the lateral transport. This is a simple coincidence, and one may as well state that the exobase distribution is controlled by one physical process or by the other. Nevertheless, in the case of no coincidence, the first physical process will induce a different ratio. The net ballistic flux will then be nonzero, producing a net lateral transport. This lateral flow will then bring back this ratio as close as possible from 
the 1.7 ratio. This is why, even with such a coincidence, it is more precise to say that the zero net ballistic flux condition controls the exobase density.

Furthermore, as is shown in Figure 20, this coincidence seems to exist for all solar activities observed, showing that the control of the exobase distribution by the Wallace and Strobel model corrected for the proton flux effect is certainly not very probable.

Returning to Figure 19, which compares $H_{\max }$ and $H_{\min }$ to the Kockarts and Nicolet [1963] model, and keeping in mind that the zero net ballistic flux condition controls the relative hydrogen density distribution, we can make the following statement: The Kockarts and Nicolet model presents a high density at night and a low density in the day, with roughly a ratio of 5 between the values. As was calculated by McAfee [1967], a density ratio of 5 would induce a strong outgoing net ballistic flux at night and a strong incoming net ballistic flux during the day. In other words, a net lateral transport of atomic hydrogen would appear from the nightside toward the dayside, decreasing the nightside density and increasing the dayside density (as defined by the Kockarts and Nicolet model). This process would last until zero net ballistic flux was reached. We would expect then to find a nighttime value of $H_{\max }$ smaller than the corresponding Kockarts and Nicolet model estimation, and a daytime value of $H_{\mathbf{m} \mid \mathbf{n}}$ greater than the corresponding Kockarts and Nicolet model estimation.

The results presented, then, in Figure 19 could be in relative agreement with the Kockarts and Nicolet model if the absolute values given by this model are increased by a factor of 2.5. This factor is quite well defined since the region where the normalized Kockarts and Nicolet model could pass (located at $1000^{\circ} \mathrm{K}$ between our measurement points) is rather small (Figure 19). Such a normalization of the Kockarts and Nicolet model would give a hydrogen density of $2.5 \times 10^{7}$ atoms $\mathrm{cm}^{-3}$ at an altitude of $100 \mathrm{~km}$. Similar values of the order

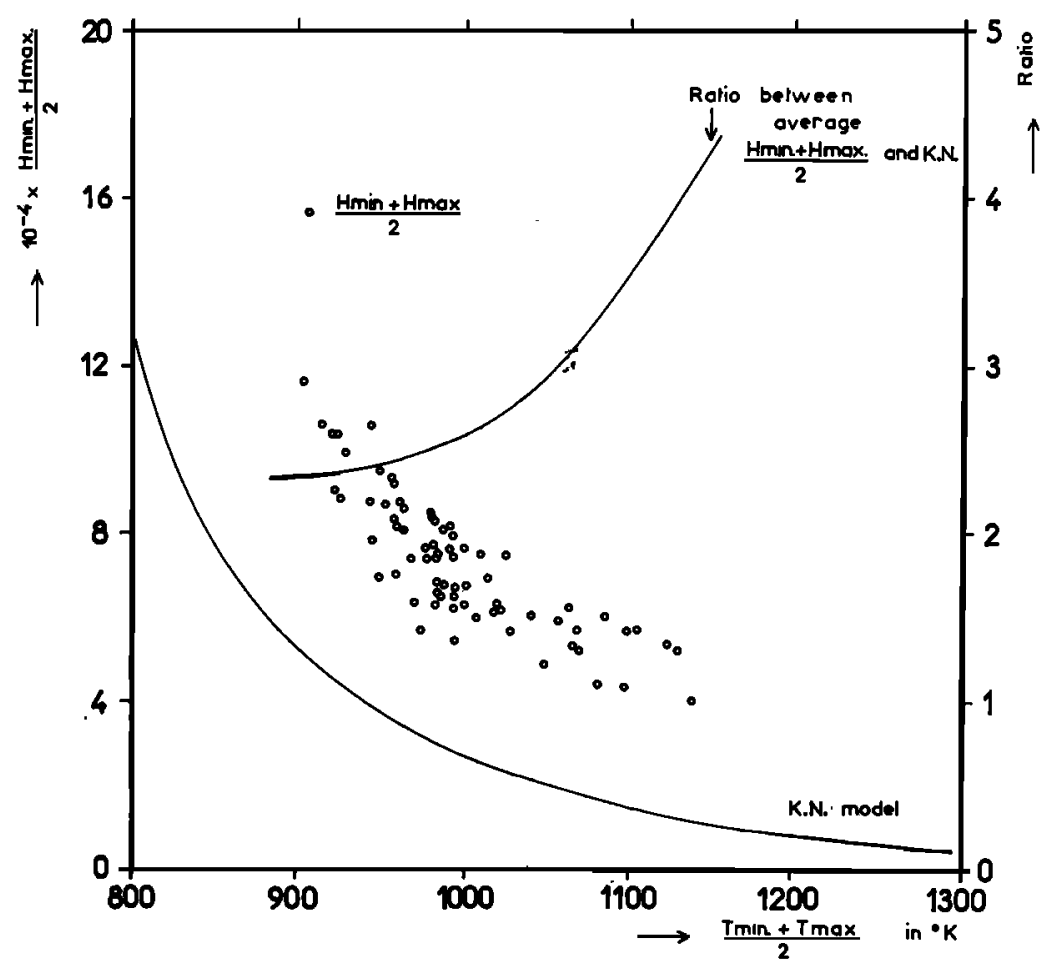

Fig. 21. Comparison of the average exobase density with the Kockarts and Nicolet model. The normalization factor of the model is represented as a function of the exospheric tem. perature. 
of $3 \times 10^{7}$ atoms $\mathrm{cm}^{-8}$ were already deduced by several authors [Donahue, 1966; Meier, 1969; Meier et al., 1970] using completely different observational techniques. Our result consequently confirms all these observations.

In order to obtain a better comparison of our data with the Kockarts and Nicolet model, which represents rather an average thermospheric situation, the average hydrogen density obtained in our study was plotted as a function of the average temperature (Figure 21). In fact, this average density, which corresponds to the exobase points located just between the minimum and maximum density points, is certainly not very much affected by the lateral transport of atomic hydrogen in the exosphere. Comparing this value with the Kockarts and Nicolet model (Figure 21), we again see that this model underestimates the hydrogen density at $500 \mathrm{~km}$. The ratio between the Kockarts and Nicolet model and the average line passing through the data points is plotted on the same figure, showing that the discrepancy increases with increasing solar activity. Again, a normalization of the Kockarts and Nicolet model might suppress all discrepancies with our data. Such a modification of the model would induce, at an altitude of $100 \mathrm{~km}$, hydrogen densities varying from $2.3 \times 10^{7}$ atoms $\mathrm{cm}^{-3}$ for low solar activity to $4 \times 10^{7}$ atoms $\mathrm{cm}^{-3}$ for high solar activity.

These values are important, because they may give an experimental indication of the influence of solar activity on the hydrogen source at an altitude of $100 \mathrm{~km}$. Nevertheless, as Shimazali and Laird [1972] pointed out, only a photochemical study of the hydrogen source, in conjunction with an estimation of the escape flux and ballistic motion effects, could give an accurate evaluation of the hydrogen density distribution in the lower thermosphere.

\section{Conchusion}

The study of the data from the experiment, conducted on board the Oso 5 spacecraft by the University of Paris, gave the following main results:

Solar Lyman $\alpha$ line. The total solar flux is of the order of $3 \times 10^{11} \mathrm{ph} \mathrm{cm}^{-2} \mathrm{sec}^{-1}$ and may reach a $30 \%$ variation during a solar rotation, best correlated with the Zurich sunspot number
$(R z)$. The flux at the line's center is of the order

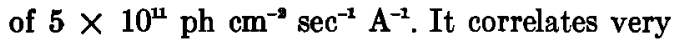
well (correlation coefficient of 0.87 ) with the total solar flux and may reach a $47 \%$ variation during a solar rotation.

Earth's hydrogen geocorona. The distribution of hydrogen from 100 to $500 \mathrm{~km}$ is certainly well represented by the Kockarts and Nicolet model [1963] with the following normalized densities at $100 \mathrm{~km}$ :

$2.3 \times 10^{7}$ atoms $\mathrm{cm}^{-3}$ for low solar activity $(R z \sim 60)$.

$2.5 \times 10^{7}$ atoms $\mathrm{cm}^{-9}$ for mean solar activity $(R z \sim 120)$.

$4 \times 10^{7}$ atoms $\mathrm{cm}^{-8}$ for high solar activity $(R z \sim 180)$.

The hydrogen density at the exobase is controlled by the zero net ballistic flux condition. The average diurnal variation of hydrogen density is of the order of a factor of 1.7. This value changes with solar activity but stays between 1.4 and 2. The data are consistent with the exospheric temperature model of Jacchia [1971]. The ratio of 1.3 between $T_{\max }$ and $T_{\min }$ is confirmed. The only difference is that the maximum temperature seems to occur a little later (1600) than in Jacchia's model. Nevertheless, this could be solely a density effect.

Acknowledgments. We would like to thank all the persons who helped us in the accomplishment of this study, particularly J. A. Quessette for very fruitful discussions. It is also a pleasure to thank the entire Oso, NASA project office, as well as the entire BBRC Oso team, who were very helpful during the long preparations of the experiment and during the entire spacecraft's life (still operating perfectly after almost 4 years in orbit). All the data processing was completed with the computer facilities of the Centre de Calcul de l'Inag. We would like to thank very much its entire operational team for their kindness and the help they so often gave to us.

The Editor thanks G. J. Kockarts and B. A. Tinsley for their assistance in evaluating this paper.

\section{REFERENCES}

Bauer, P., P. Waldteufel, and D. Alcayde, Diurnal variations of the atomic oxygen density and temperature determined from incoherent scatter measurements in the ionospheric $F$ region, $J$. Geophys. Res., 75, 4825-4832, 1970.

Blamont, J. E., and A. Vidal-Madjar, Monitoring 
of the solar Lyman-alpha line during the year 1969, J. Geophys. Res., 76, 4311-4324, 1971.

Brinton, H. C., and H. G. Mayr, Temporal variations of thermospheric hydrogen derived from in situ measurements, J. Geophys. Res., 76,6198 $6201,1971$.

Chamberlain, J. W., Planetary coronae and atmospheric evaporation, Planet. Space Sci., 11, 901, 1963.

Donahue, T. M., The problem of atomic hydrogen, Ann. Geophys., 22, 175-188, 1966.

Godart, M., and M. Nicolet, Diurnal variation of atomic hydrogen in the upper atmosphere, paper presented at the 4th International Space Science Symposium, Cospar and Pol. Acad. of Sci., Warsaw, June 4-10, 1963.

Hanson, W. B., and T. N. L. Patterson, Diurnal variation of the hydrogen concentration in the exosphere, Planet. Space Sci., 11, 1035-1052, 1963.

Ho, M. C., and D. R. Moorcroft, Hydrogen density and proton flux in the topside ionosphere over Arecibo, Puerto Rico, from incoherent scatter observations, Planet. Space Sci., 19, 1441-1445, 1971.

Jacchia, L. G., Revised static models of the thermosphere and exosphere with empirical temperature profiles, in Research in Space Science, Spec. Rep. 33\%, Smithson. Astrophys. Observ., Cambridge, Mass., 1971.

Kockarts, G., and M. Nicolet, Le problème aéronomique de l'hélium et de l'hydrogène neutre, Ann. Geophys., 19, 269, 1963.

McAfee, J. R., Lateral flow in the exosphere, Planet. Space Sci., 15, 599-609, 1967.
Meier, R. R., Temporal variations of solar Lyman alpha, J. Geophys. Res., 74, 6487-6490, 1969a.

Meier, R. R., Balmer alpha and Lyman beta in the hydrogen geocorona, J. Geophys. Res., 74, 3561-3574, $1969 b$.

Meier, R. R., D. M. Weiss, and P. Mange, High altitude measurement of the Lyman alpha night glow at solar minimum, J. Geophys. Res., 75, 4224-4229, 1970.

Metzger, P. H., and M. A. Clark, On the diurnal variation of the exospheric neutral hydrogen temperature, J. Geophys. Res., 75, 5587, 1970.

Patterson, T. N. L., The diurnal variation of atomic hydrogen concentration at the base of the exosphere, Planet. Space Sci., 14, 425, 1966.

Quesette, J. A., Atomic hydrogen densities at the exobase, J. Geophys. Res., 77, 2997-3000, 1972.

Shimazaki, T., and A. R. Laird, Seasonal effects on distributions of minor neutral constituents in the mesosphere and lower thermosphere, Radio Sci., 7, 23-43, 1972.

Tinsley, B. A., Variations of Balmer emission and related hydrogen distributions, Space Res., 10, 582-590, 1970.

Tinsley, B. A., The diumal variation of atomic hydrogen, Planet. Space Sci., in press, 1973.

Vidal-Madjar, A., and J. L. Bertaux, A calculated hydrogen distribution in the exosphere, Planet. Space Sci., 20, 1147-1162, 1972.

Wallace, L., and D. F. Strobel, Diurnal variation of atomic hydrogen in the thermosphere, Planet. Space Sci., 20, 521-531, 1972.

(Received August 1, 1972 ; accepted November 1, 1972.) 